\title{
Transition-Metal Free Mechanochemical Approach to Polyyne Substituted Pyrroles
}

Bartłomiej Pigulski ${ }^{1}$, Agata Arendt ${ }^{1}$, Denis N. Tomilin ${ }^{2}$, Lyubov N. Sobenina ${ }^{2}$, Boris A. Trofimov $^{2}$, Sławomir Szafert ${ }^{1 *}$

${ }^{1}$ Department of Chemistry, University of Wrocław, 14 F. Joliot-Curie, 50-383 Wrocław, Poland.

${ }^{2}$ A. E. Favorsky Irkutsk Institute of Chemistry, Siberian Branch, Russian Academy of Sciences, 1 Favorsky, 664033, Irkutsk, Russian Federation.

\section{Table of Contents}

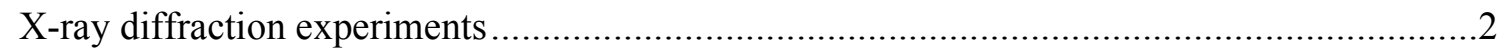

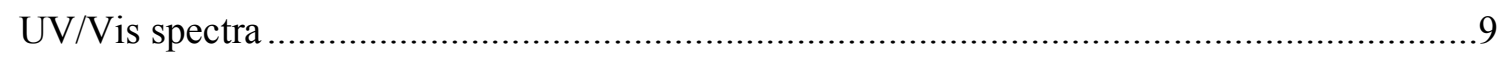

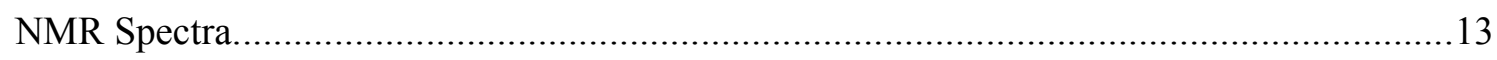




\section{X-ray diffraction experiments}

The space groups were determined from systematic absences and subsequent least-squares refinement. Lorentz and polarization corrections were applied. The structures were solved by direct methods and refined by full-matrix, least-squares on $F^{2}$ by use of the SHELXTL Package. ${ }^{1}$ Non-hydrogen atoms were refined with anisotropic thermal parameters. Hydrogen atom positions were calculated and added to the structure factor calculations, but were not refined

Table 1. Details of X-ray diffraction experiments

\begin{tabular}{|c|c|c|c|c|c|c|}
\hline & 1- $\mathrm{C}_{6} \mathrm{THI}-\mathrm{Me}$ & 2- $\mathrm{C}_{6}$ THI-Vin & 2- $\mathrm{C}_{6} \mathrm{THI}-\mathrm{Bn}$ & 3- $\mathrm{C}_{6}$ THI-Vin & 4- $\mathrm{C}_{6} \mathrm{THI}-\mathrm{Bn}$ & 2- $\mathrm{C}_{8}$ THI-Vin \\
\hline Chemical formula & $\mathrm{C}_{22} \mathrm{H}_{16} \mathrm{~N}_{2}$ & $\mathrm{C}_{22} \mathrm{H}_{16} \mathrm{~N}_{2} \mathrm{O}_{2}$ & $\mathrm{C}_{27} \mathrm{H}_{20} \mathrm{~N}_{2} \mathrm{O}_{2}$ & $\mathrm{C}_{24} \mathrm{H}_{19} \mathrm{NO}$ & $\mathrm{C}_{30} \mathrm{H}_{25} \mathrm{NO}_{2}$ & $\mathrm{C}_{24} \mathrm{H}_{16} \mathrm{~N}_{2} \mathrm{O}_{2}$ \\
\hline$M_{\mathrm{r}}$ & 308.37 & 340.37 & 404.45 & 337.40 & 431.51 & 364.39 \\
\hline $\begin{array}{l}\text { Crystal system, } \\
\text { space group }\end{array}$ & Triclinic, $P-1$ & Triclinic, $P-1$ & Monoclinic, $C 2 / c$ & Triclinic, $P-1$ & Triclinic, $P-1$ & Monoclinic, $P 2_{1} / c$ \\
\hline Temperature (K) & 100 & 100 & 100 & 100 & 150 & 100 \\
\hline$a, b, c(\AA)$ & $\begin{array}{l}7.361(2), 7.922 \\
(2), 15.443(5)\end{array}$ & $\begin{array}{l}7.011(2), 8.377 \\
(2), 15.363(5)\end{array}$ & $\begin{array}{l}19.946(6), 13.024 \\
(4), 18.084(6)\end{array}$ & $\begin{array}{l}7.284(2), 9.238 \\
(3), 14.084(5)\end{array}$ & $\begin{array}{l}7.668(3), 11.842 \\
(4), 13.298(5)\end{array}$ & $\begin{array}{l}15.996(5), 6.903 \\
(2), 17.091(6)\end{array}$ \\
\hline$\alpha, \beta, \gamma\left({ }^{\circ}\right)$ & $\begin{array}{l}93.97(3), 103.51 \\
(3), 107.90(3)\end{array}$ & $\begin{array}{l}100.03(3), 94.52 \\
(3), 105.83(3)\end{array}$ & $90,120.95(3), 90$ & $\begin{array}{l}97.60(3), 91.20 \\
(3), 109.80(4)\end{array}$ & $\begin{array}{l}92.89(3), 93.77 \\
(3), 104.73(4)\end{array}$ & $90,105.90(3), 90$ \\
\hline$V\left(\AA^{3}\right)$ & $823.5(4)$ & $847.2(4)$ & 4029 (2) & $881.6(5)$ & $1162.5(8)$ & $1815.0(10)$ \\
\hline$Z$ & 2 & 2 & 8 & 2 & 2 & 4 \\
\hline Radiation type & Mo $K \alpha$ & Mo $K \alpha$ & Mo $K \alpha$ & $\mathrm{Cu} K \alpha$ & Mo $K \alpha$ & $\mathrm{Cu} K \alpha$ \\
\hline$\mu\left(\mathrm{mm}^{-1}\right)$ & 0.07 & 0.09 & 0.09 & 0.60 & 0.08 & 0.69 \\
\hline Crystal size (mm) & $0.40 \times 0.20 \times 0.04$ & $0.39 \times 0.33 \times 0.07$ & $0.47 \times 0.32 \times 0.11$ & $0.20 \times 0.12 \times 0.03$ & $0.44 \times 0.40 \times 0.31$ & $0.41 \times 0.15 \times 0.04$ \\
\hline $\begin{array}{l}\text { Absorption } \\
\text { correction }\end{array}$ & - & - & - & - & - & - \\
\hline $\begin{array}{l}\text { No. of measured, } \\
\text { independent and } \\
\text { observed }[I> \\
2 \sigma(I)] \text { reflections }\end{array}$ & $7476,3575,1949$ & $5810,3637,1389$ & $14387,3954,3251$ & $4652,2776,904$ & $13755,7938,5320$ & $5088,2844,1634$ \\
\hline$R_{\text {int }}$ & 0.031 & 0.084 & 0.032 & 0.095 & 0.045 & 0.058 \\
\hline$(\sin \theta / \lambda)_{\max }\left(\AA^{-1}\right)$ & 0.639 & 0.838 & 0.617 & 0.580 & 0.843 & 0.580 \\
\hline $\begin{array}{l}R\left[F^{2}>\right. \\
\left.2 \sigma\left(F^{2}\right)\right], w R\left(F^{2}\right), S\end{array}$ & $0.061,0.163,0.90$ & $0.075,0.176,0.85$ & $0.044,0.129,1.11$ & $0.075,0.158,0.93$ & $0.072,0.211,1.03$ & $0.067,0.185,0.88$ \\
\hline No. of reflections & 3575 & 3637 & 3954 & 2776 & 7938 & 2844 \\
\hline No. of parameters & 303 & 235 & 280 & 236 & 367 & 253 \\
\hline No. of restraints & 27 & 0 & 0 & 0 & 19 & 0 \\
\hline $\begin{array}{l}\Delta \rho_{\max }, \Delta \rho_{\min }(\mathrm{e} \\
\left.\AA^{-3}\right)\end{array}$ & $0.33,-0.20$ & $0.28,-0.25$ & $0.29,-0.27$ & $0.32,-0.27$ & $0.48,-0.27$ & $0.42,-0.30$ \\
\hline
\end{tabular}

\footnotetext{
${ }^{1}$ Sheldrick, G. M. Acta Crystallograph. A 2008, 64, 112-122.
} 


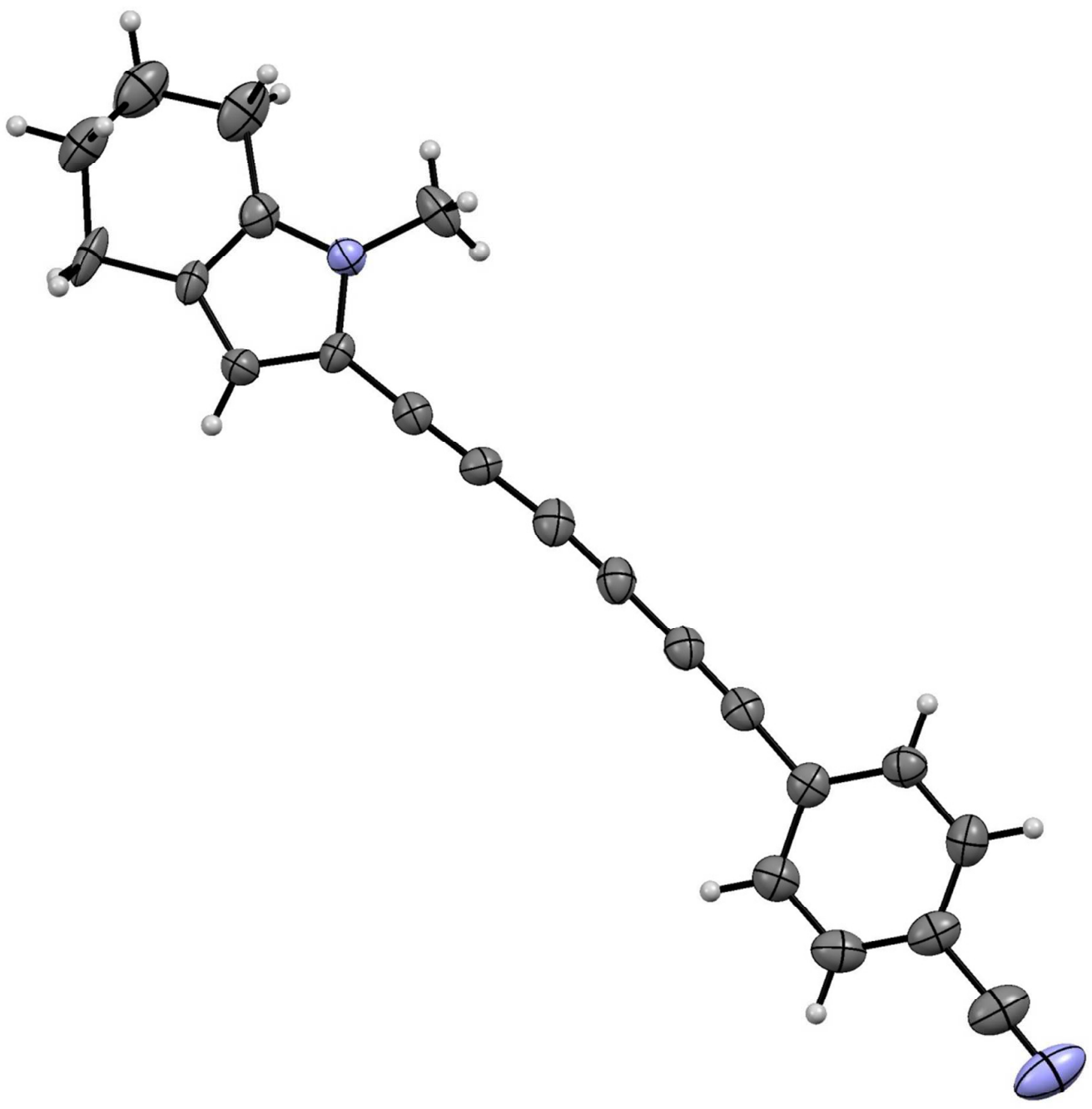

Figure 1. Molecular structure of $1-\mathrm{C}_{6} \mathrm{THI}-\mathrm{Me}$ (main conformation). Thermal ellipsoids are given at $50 \%$ probability level. Disorder is omitted for clarity. 


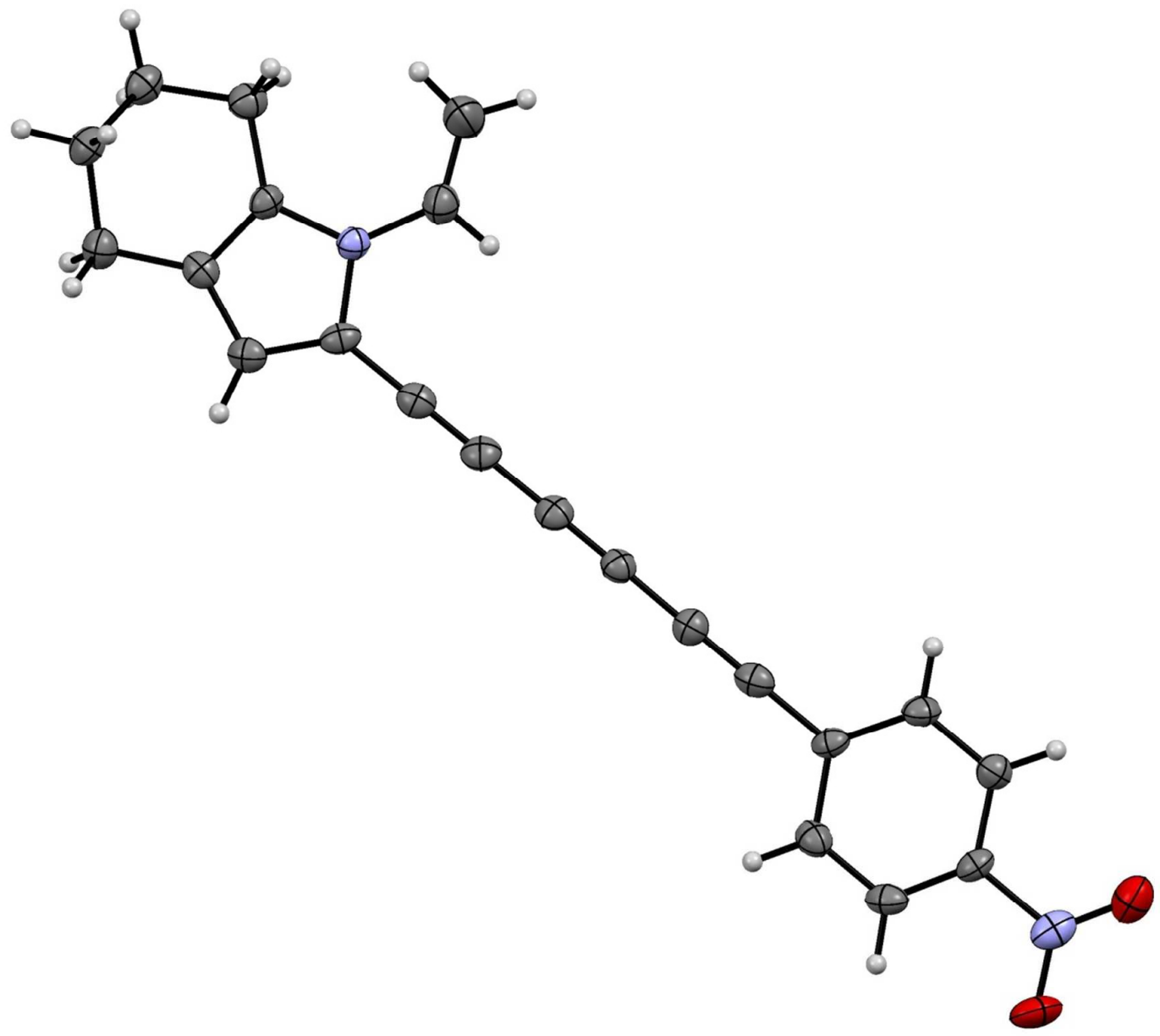

Figure 2. Molecular structure of $1-\mathrm{C}_{6} \mathrm{THI}-\mathrm{Vin}$. Thermal ellipsoids are given at $50 \%$ probability level. 


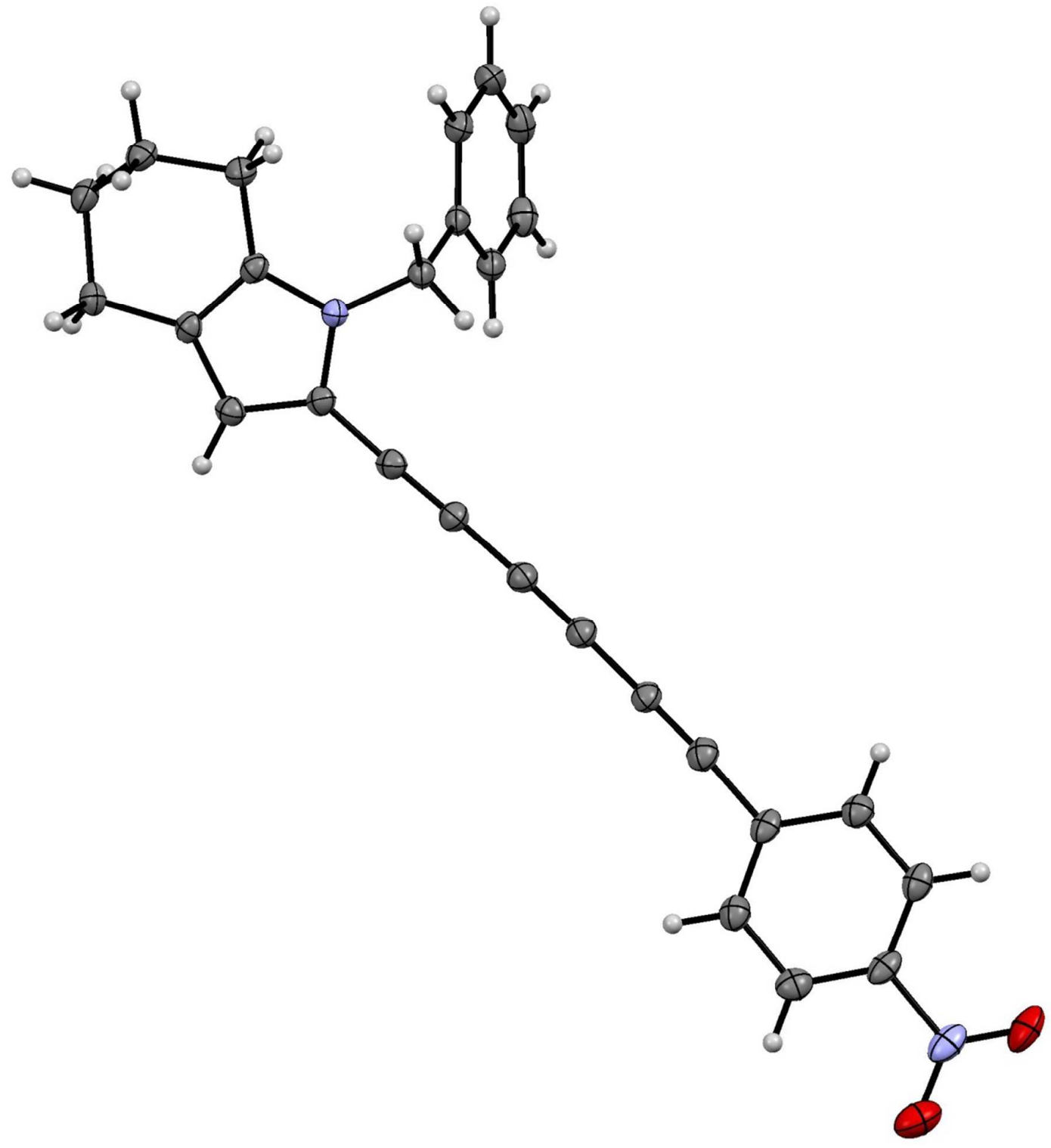

Figure 3. Molecular structure of $1-\mathrm{C}_{6}$ THI-Bn. Thermal ellipsoids are given at $50 \%$ probability level. 


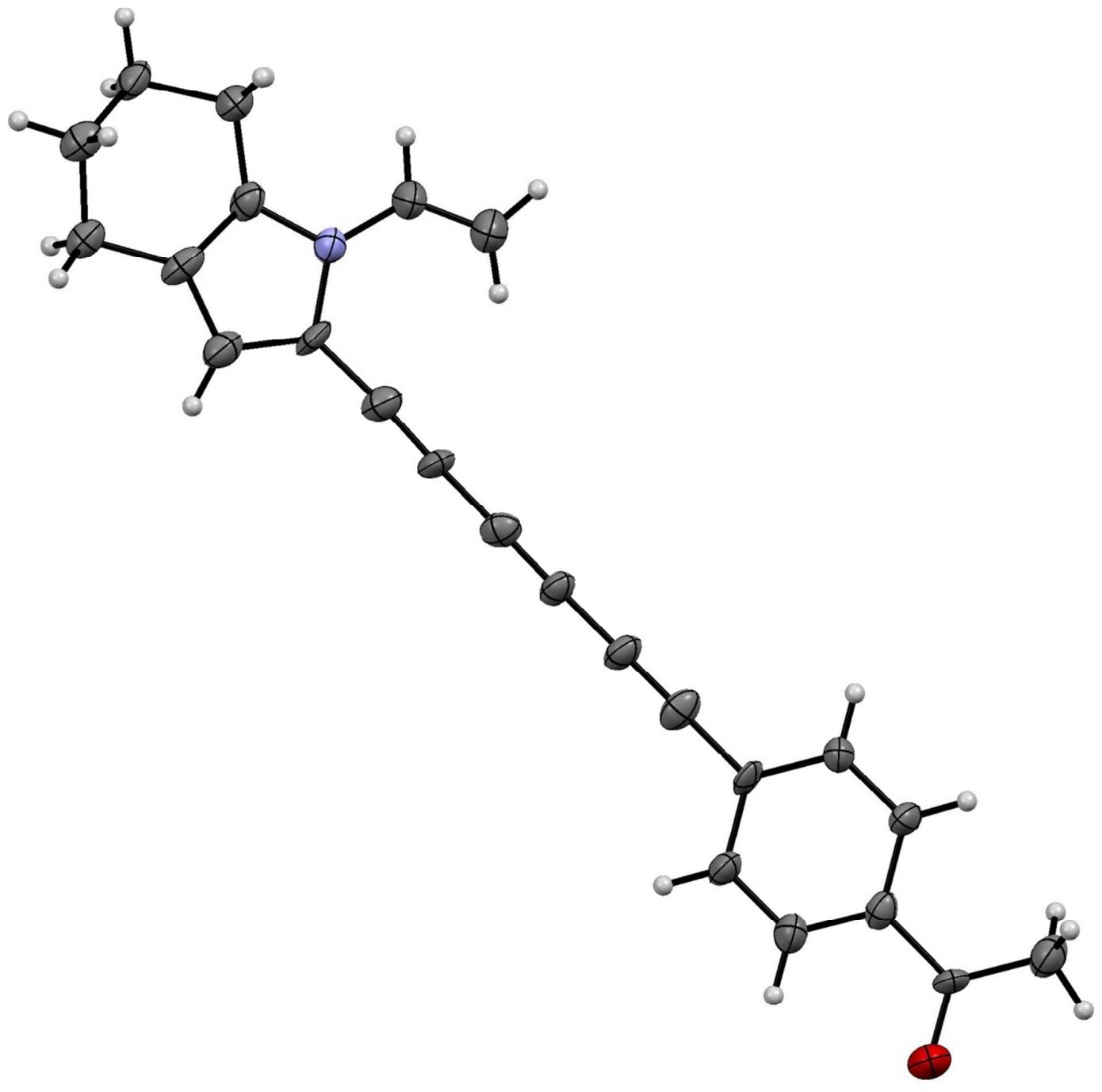

Figure 4. Molecular structure of $3-\mathrm{C}_{6} \mathrm{THI}-\mathrm{Vin}$. Thermal ellipsoids are given at 50\% probability level. 


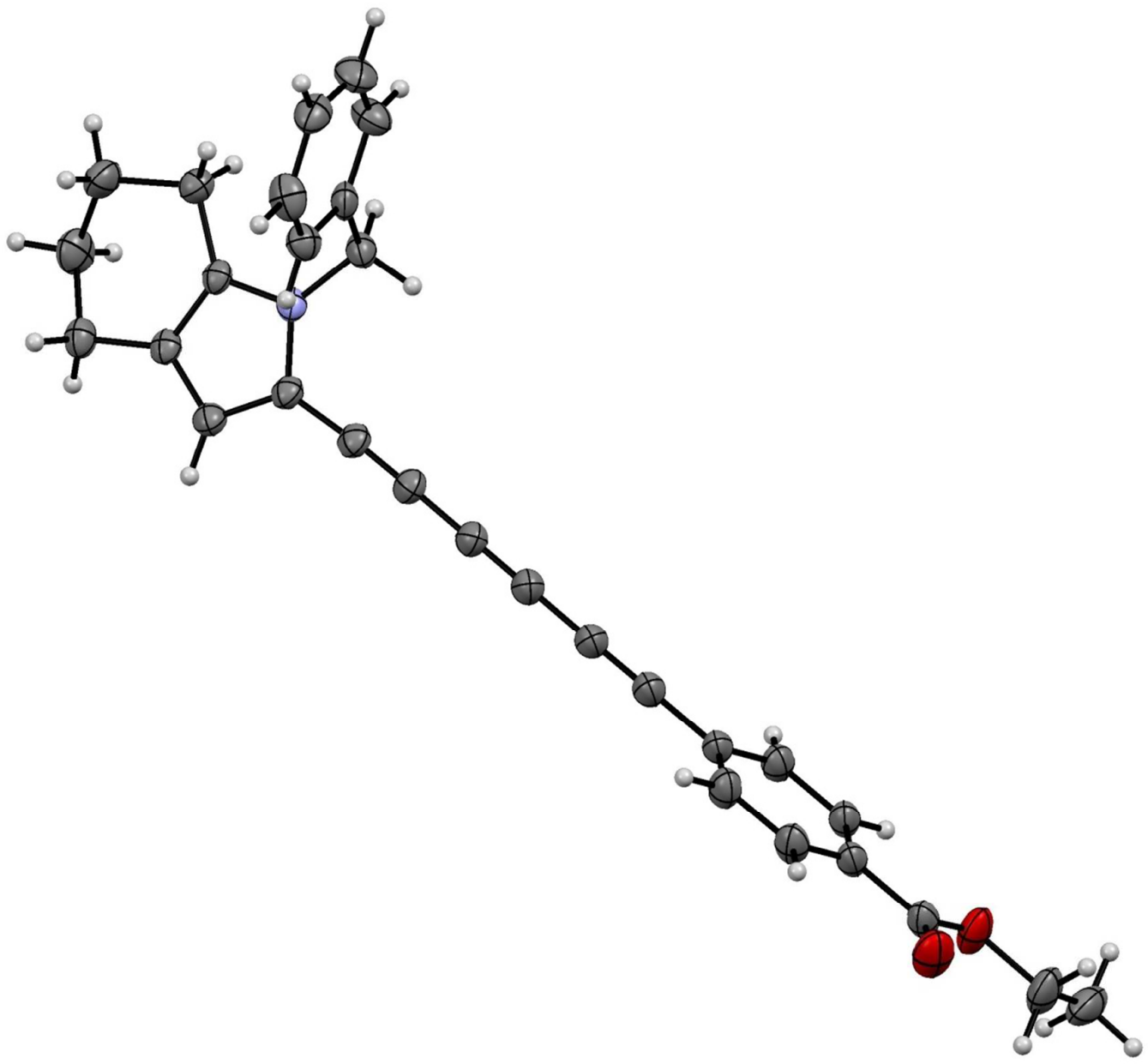

Figure 5. Molecular structure of 4- $\mathrm{C}_{6}$ THI-Bn (main conformation). Thermal ellipsoids are given at $50 \%$ probability level. Disorder is omitted for clarity. 


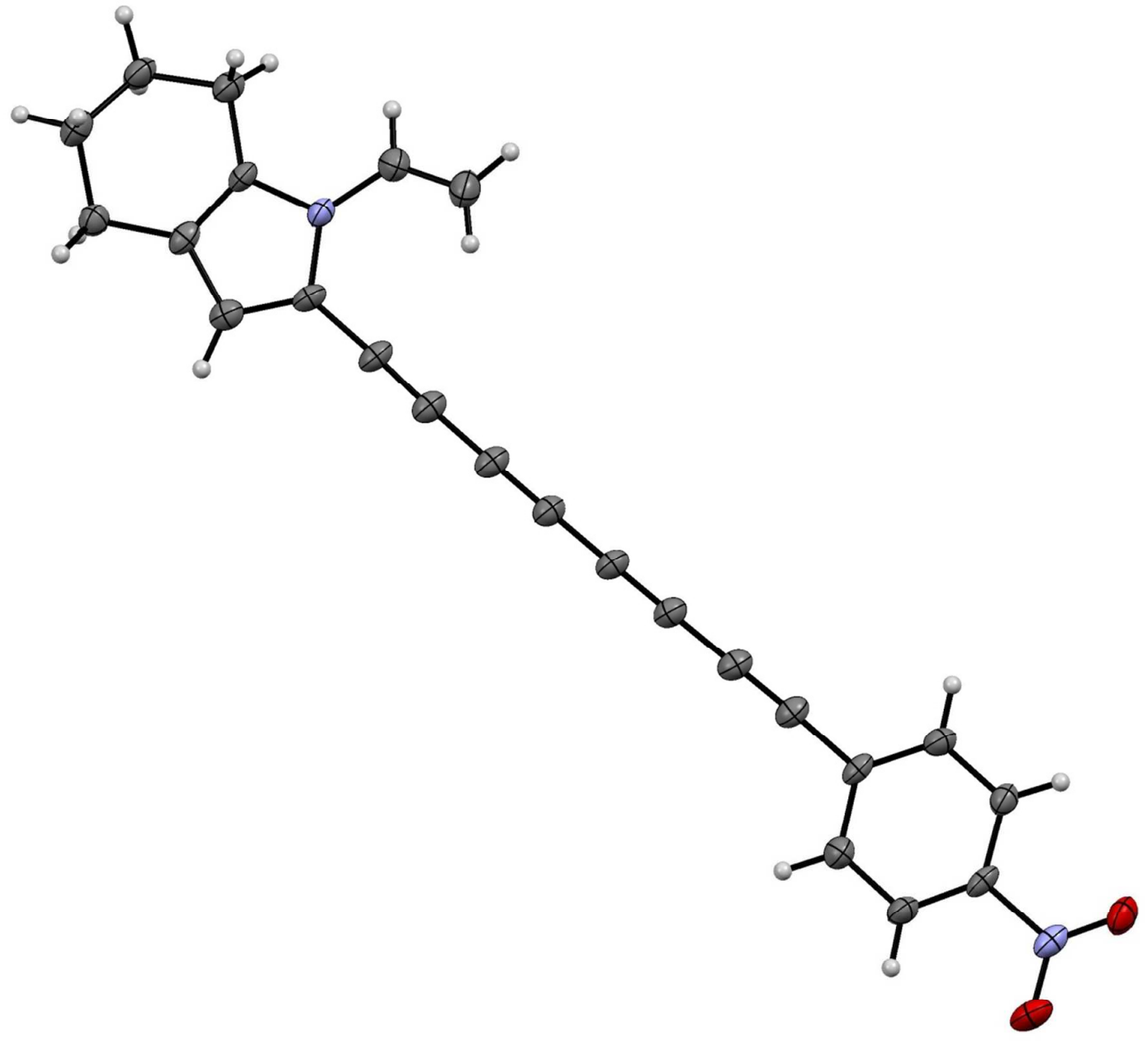

Figure 6. Molecular structure of $2-\mathrm{C}_{8} \mathrm{THI}-\mathrm{Vin}$. Thermal ellipsoids are given at $50 \%$ probability level. 


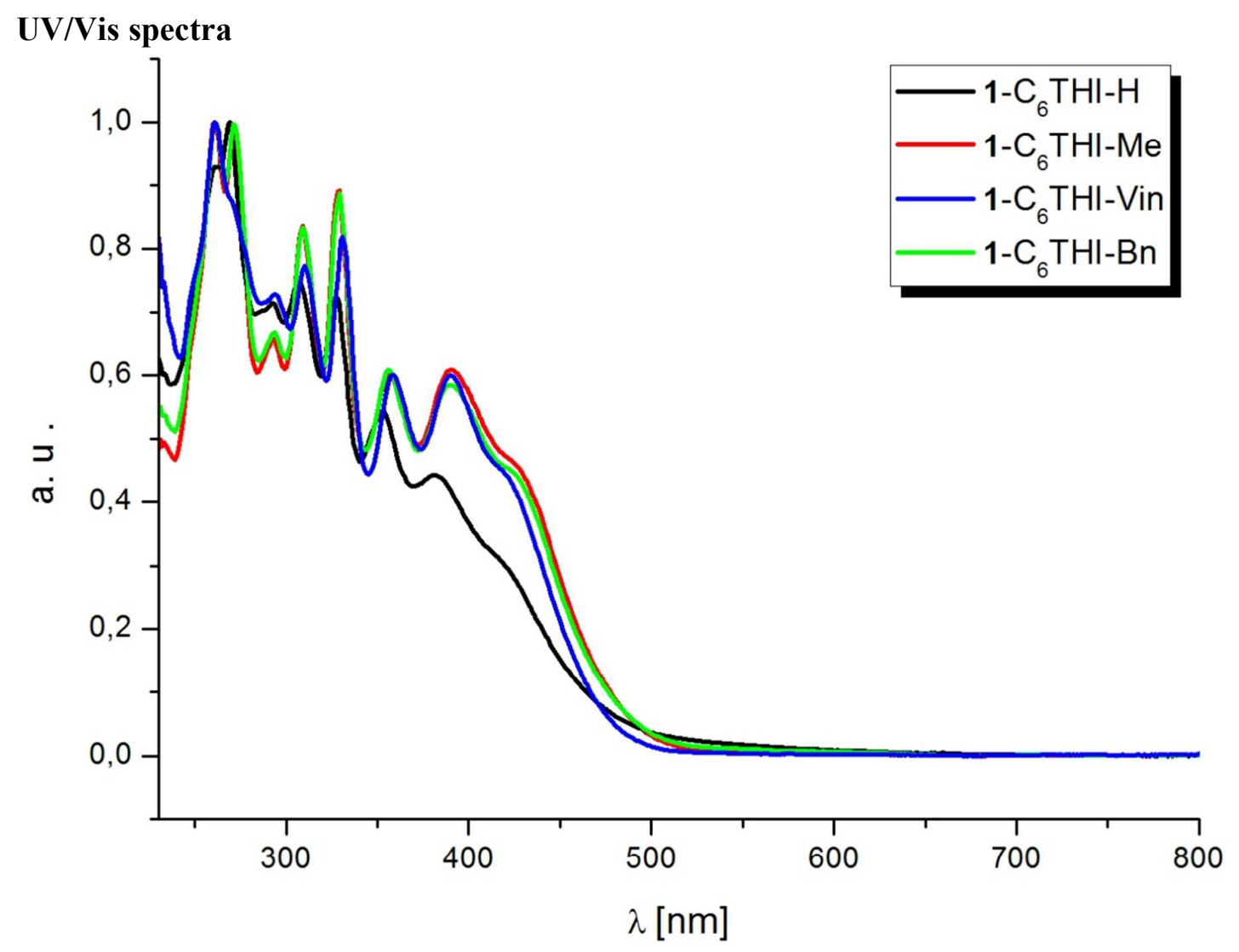

Figure 7. UV/Vis spectra of $1-\mathrm{C}_{6}$ THI-H, $1-\mathrm{C}_{6}$ THI-Me. $1-\mathrm{C}_{6}$ THI-Vin and $1-\mathrm{C}_{6} \mathrm{THI}-\mathrm{Bn}$ $\left(\mathrm{CH}_{2} \mathrm{Cl}_{2}, 298 \mathrm{~K}\right)$.

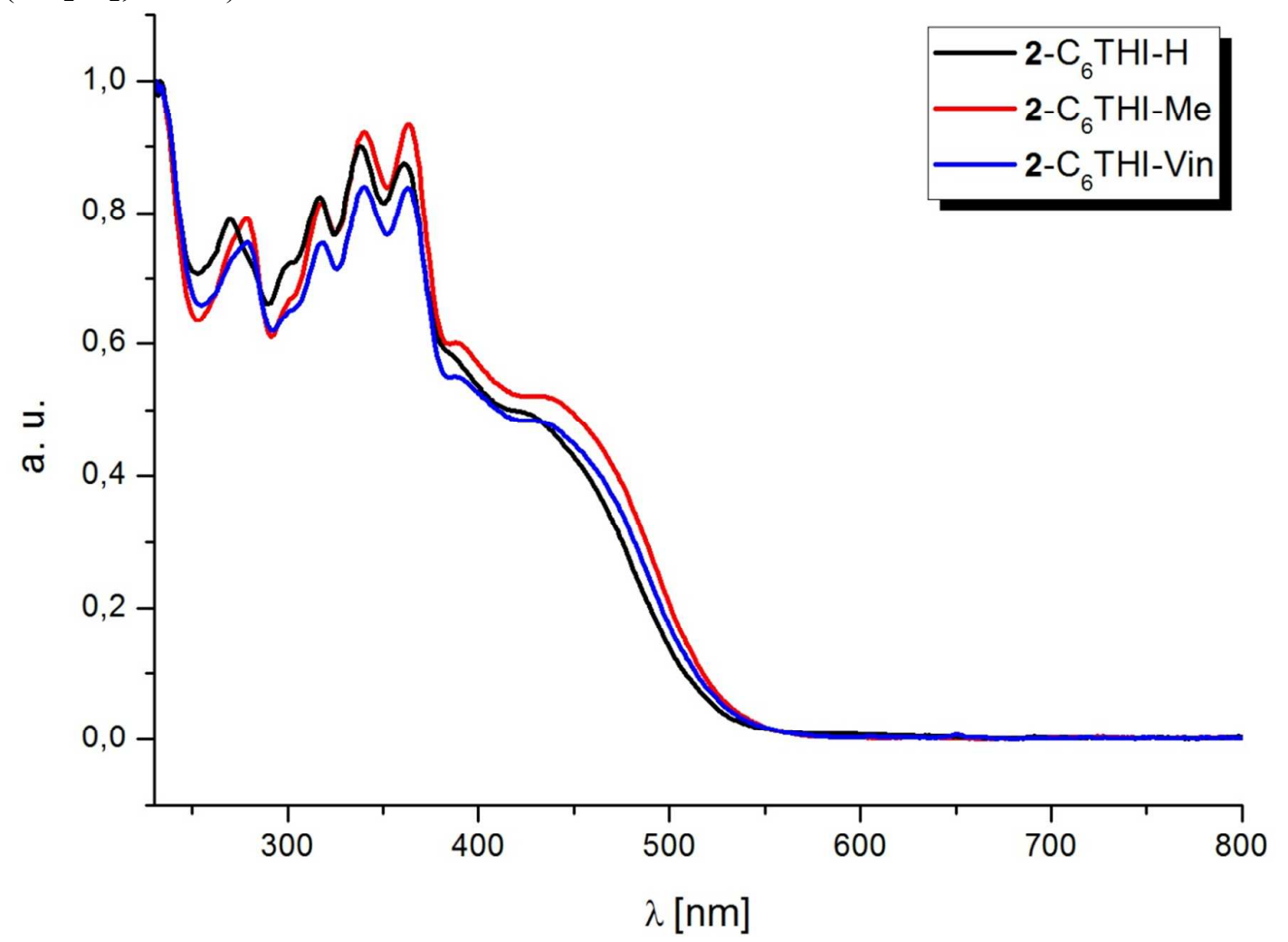

Figure 8. UV/Vis spectra of 2- $\mathrm{C}_{6} \mathrm{THI}-\mathrm{H}, 2-\mathrm{C}_{6} \mathrm{THI}-\mathrm{Me}$ and 2- $\mathrm{C}_{6} \mathrm{THI}-\mathrm{Bn}\left(\mathrm{CH}_{2} \mathrm{Cl}_{2}, 298 \mathrm{~K}\right)$. 


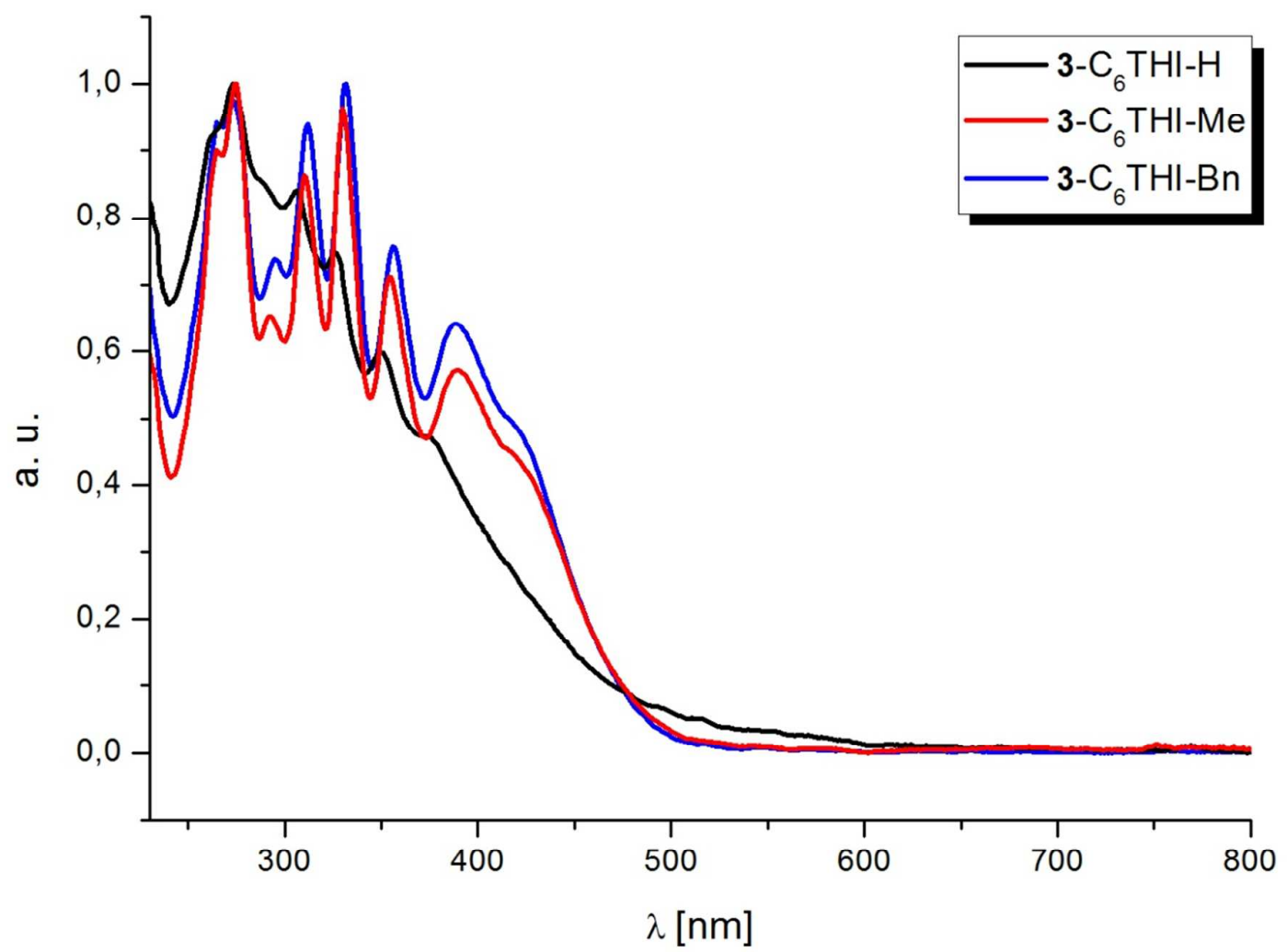

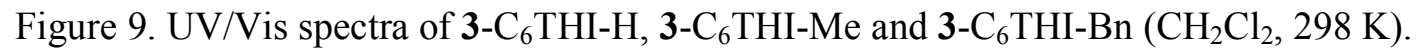

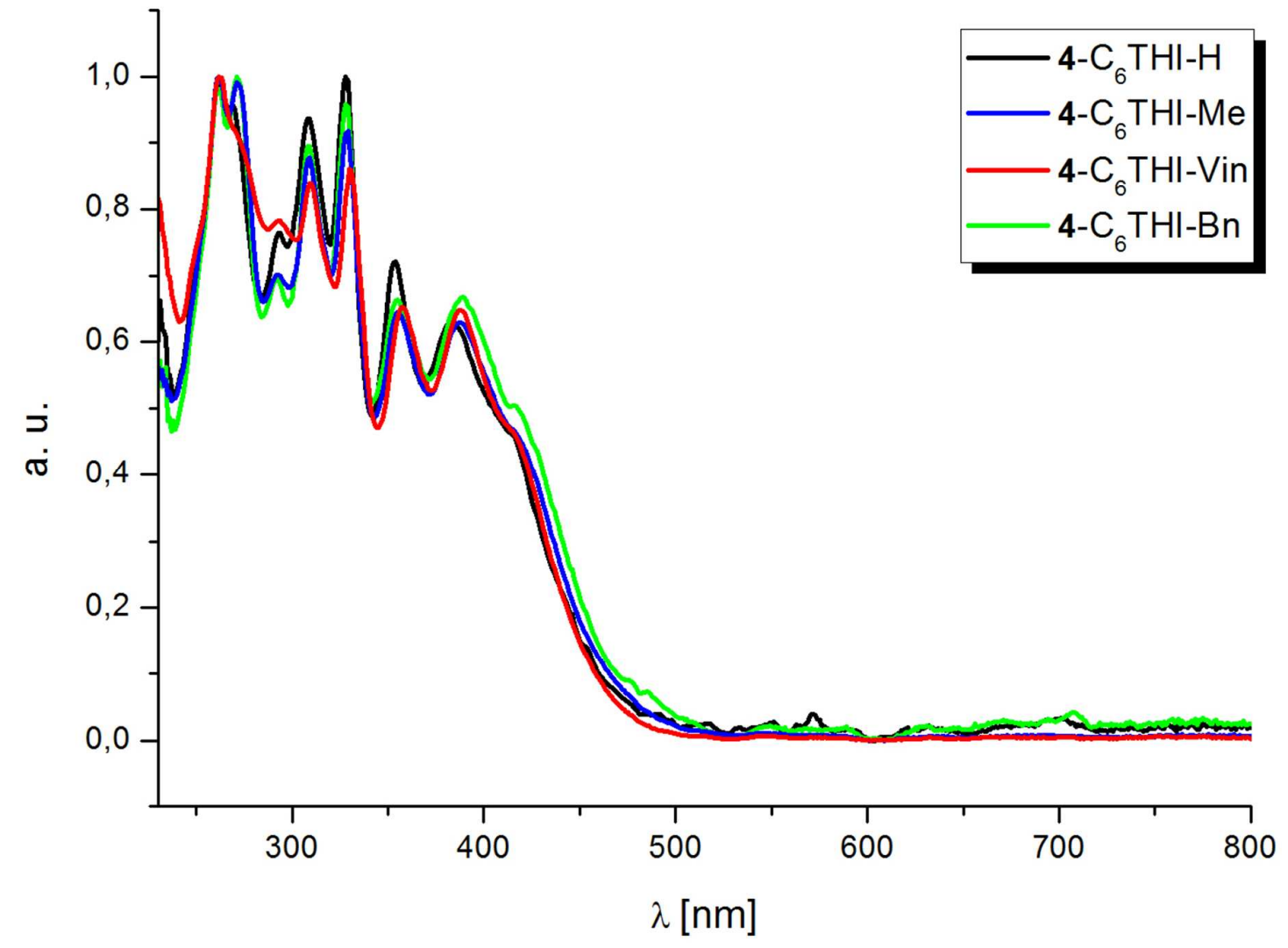

Figure 10. UV/Vis spectra of 4- $\mathrm{C}_{6}$ THI-H, 4-C 6 THI-Me, 4- $\mathrm{C}_{6} \mathrm{THI}-\mathrm{Vin}$ and $4-\mathrm{C}_{6} \mathrm{THI}-\mathrm{Bn}$ $\left(\mathrm{CH}_{2} \mathrm{Cl}_{2}, 298 \mathrm{~K}\right)$. 


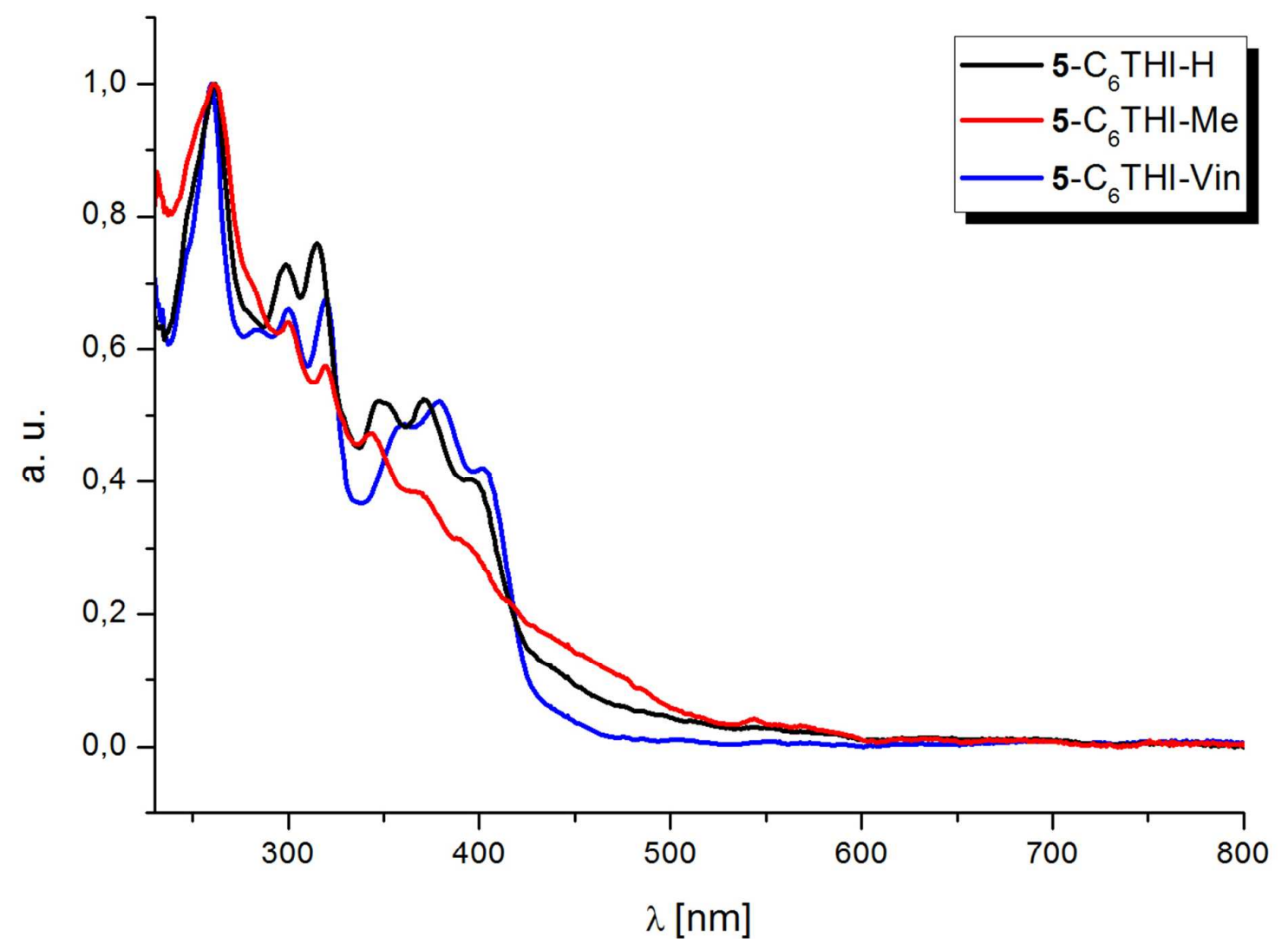

Figure 11. UV/Vis spectra of 5- $\mathrm{C}_{6} \mathrm{THI}-\mathrm{H}, 5-\mathrm{C}_{6} \mathrm{THI}-\mathrm{Me}$ and 5- $\mathrm{C}_{6} \mathrm{THI}-\mathrm{Vin}\left(\mathrm{CH}_{2} \mathrm{Cl}_{2}, 298 \mathrm{~K}\right)$.

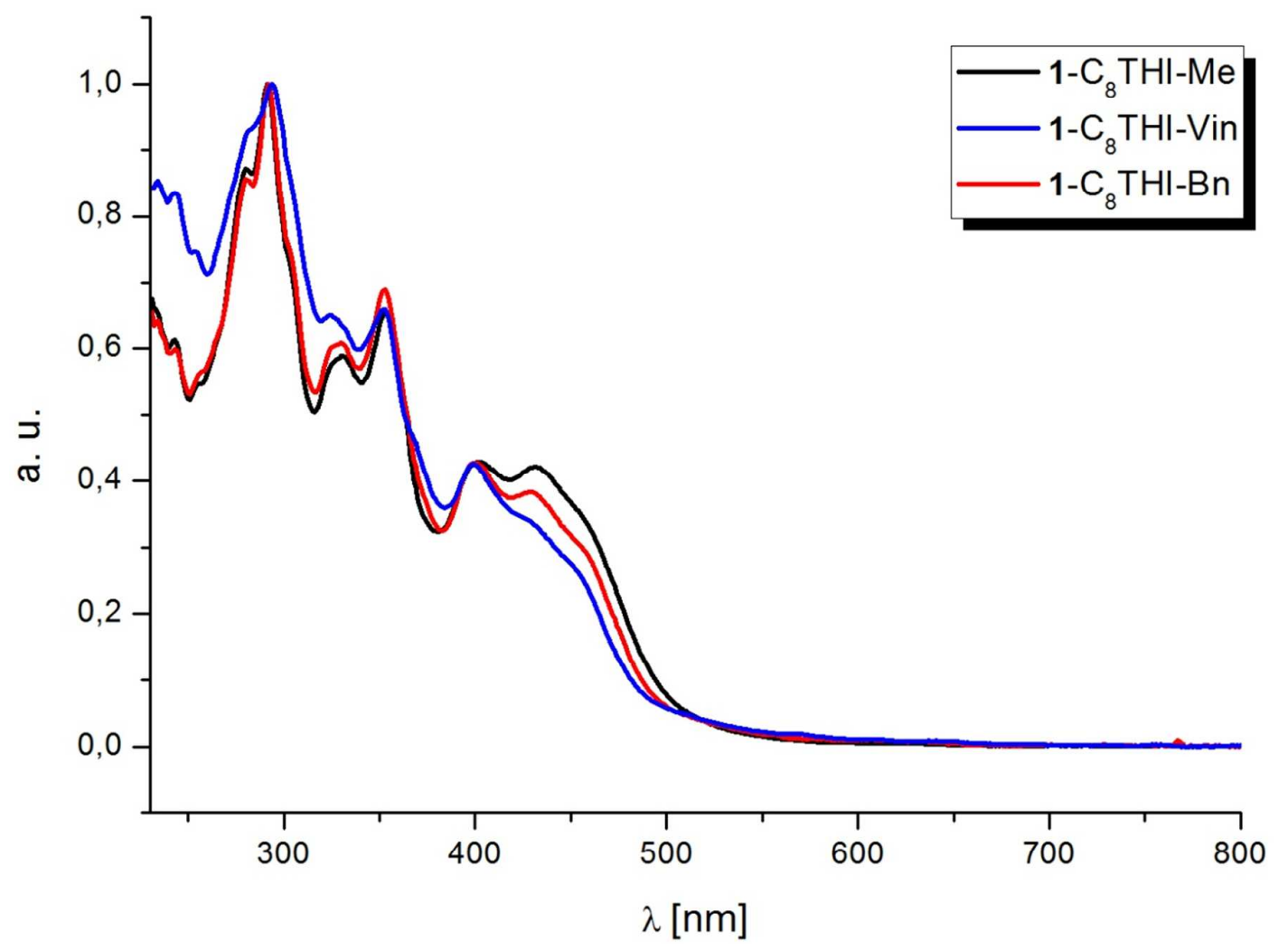

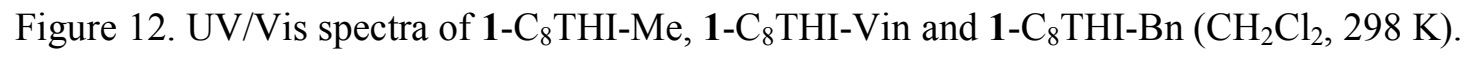




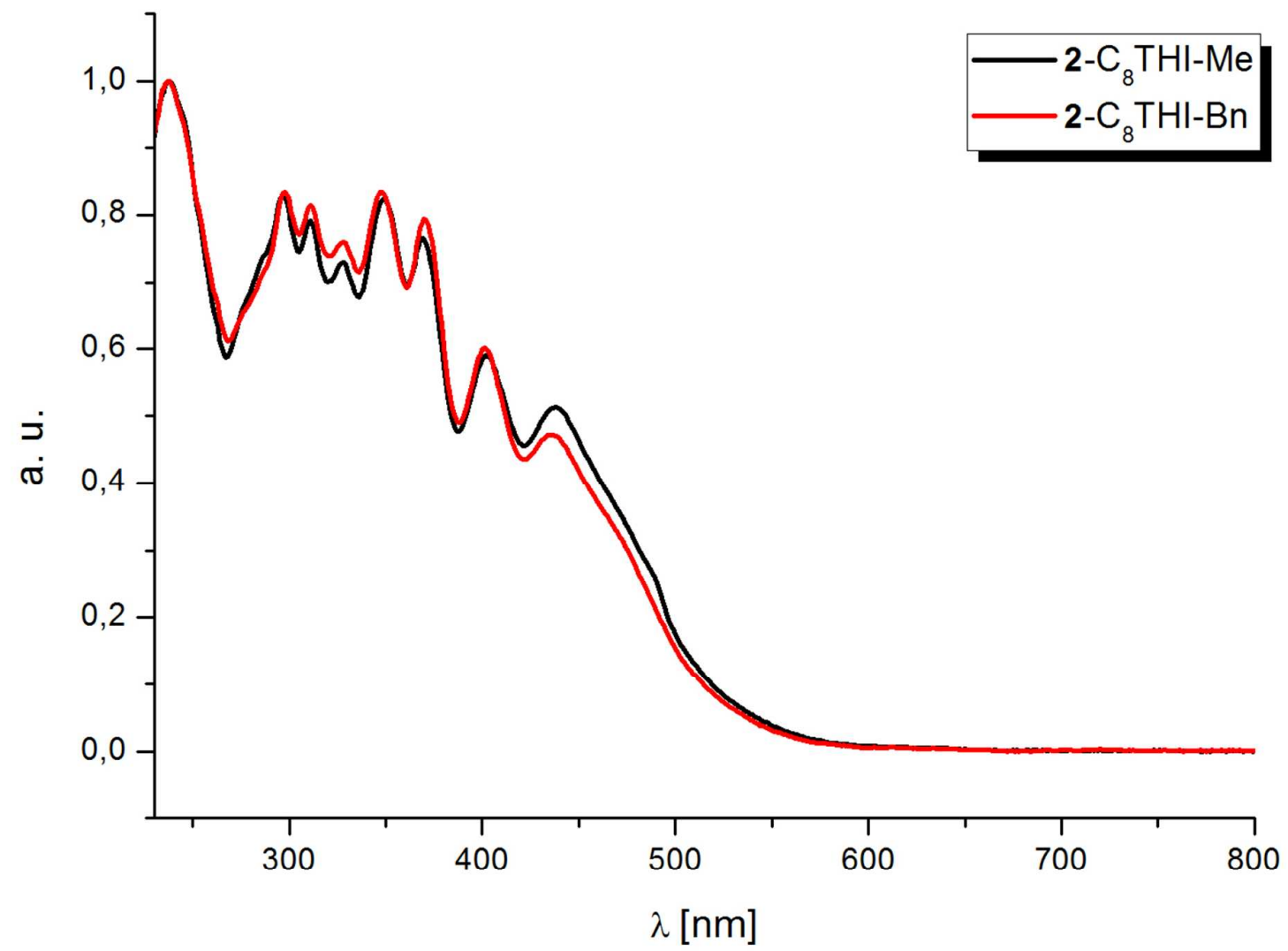

Figure 13. UV/Vis spectra of 2- $\mathrm{C}_{8} \mathrm{THI}-\mathrm{Me}$ and 2-C $\mathrm{CHI}-\mathrm{Bn}\left(\mathrm{CH}_{2} \mathrm{Cl}_{2}, 298 \mathrm{~K}\right)$. 


\section{NMR Spectra}

\section{(3-C 6 TMS) 1-(4-((Trimethylsilyl)hexatriynyl)phenyl)ethanone}
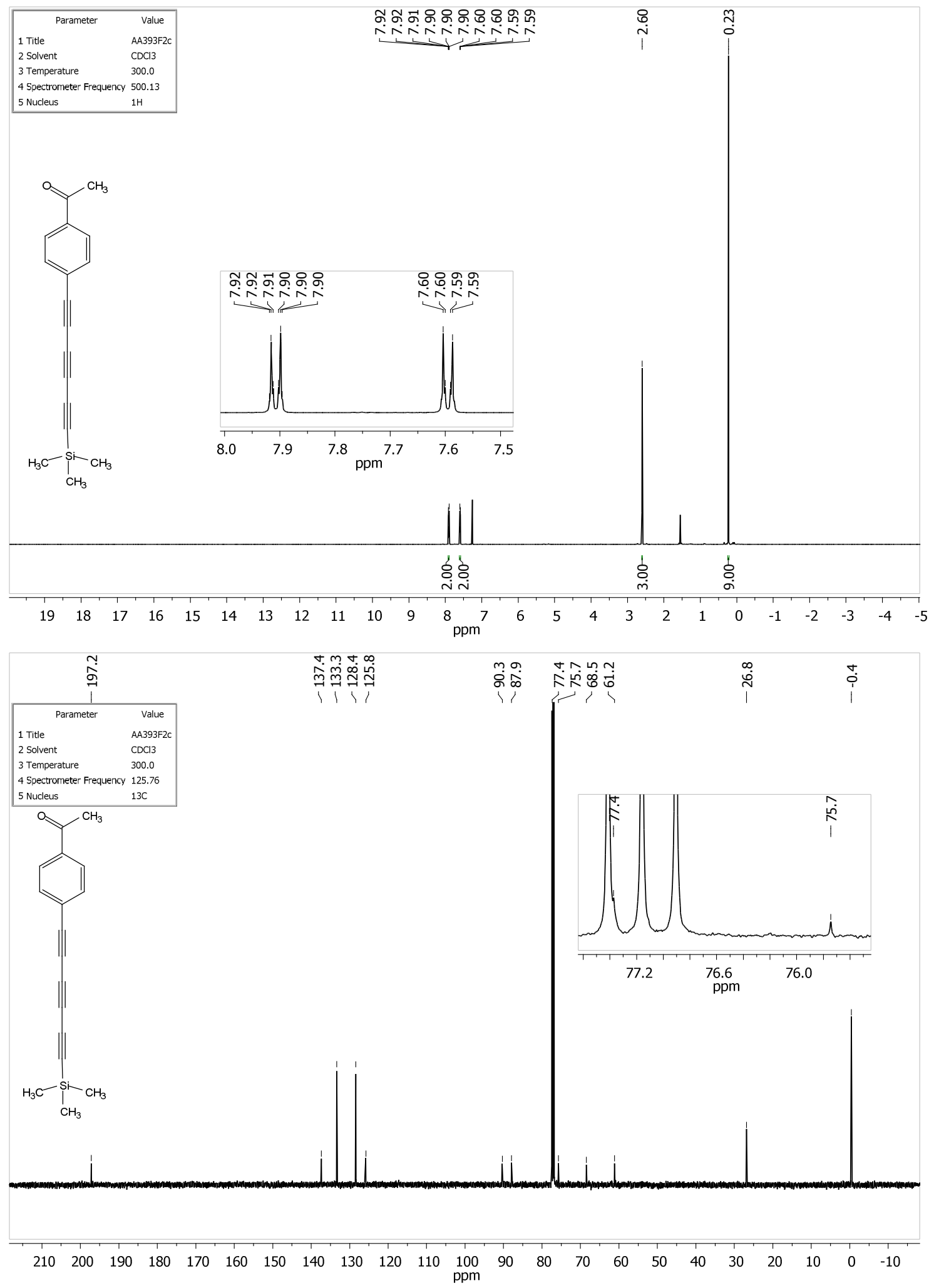


\section{(4-C 6 TMS) Ethyl 4-((trimethylsilyl)hexatriynyl)benzoate}
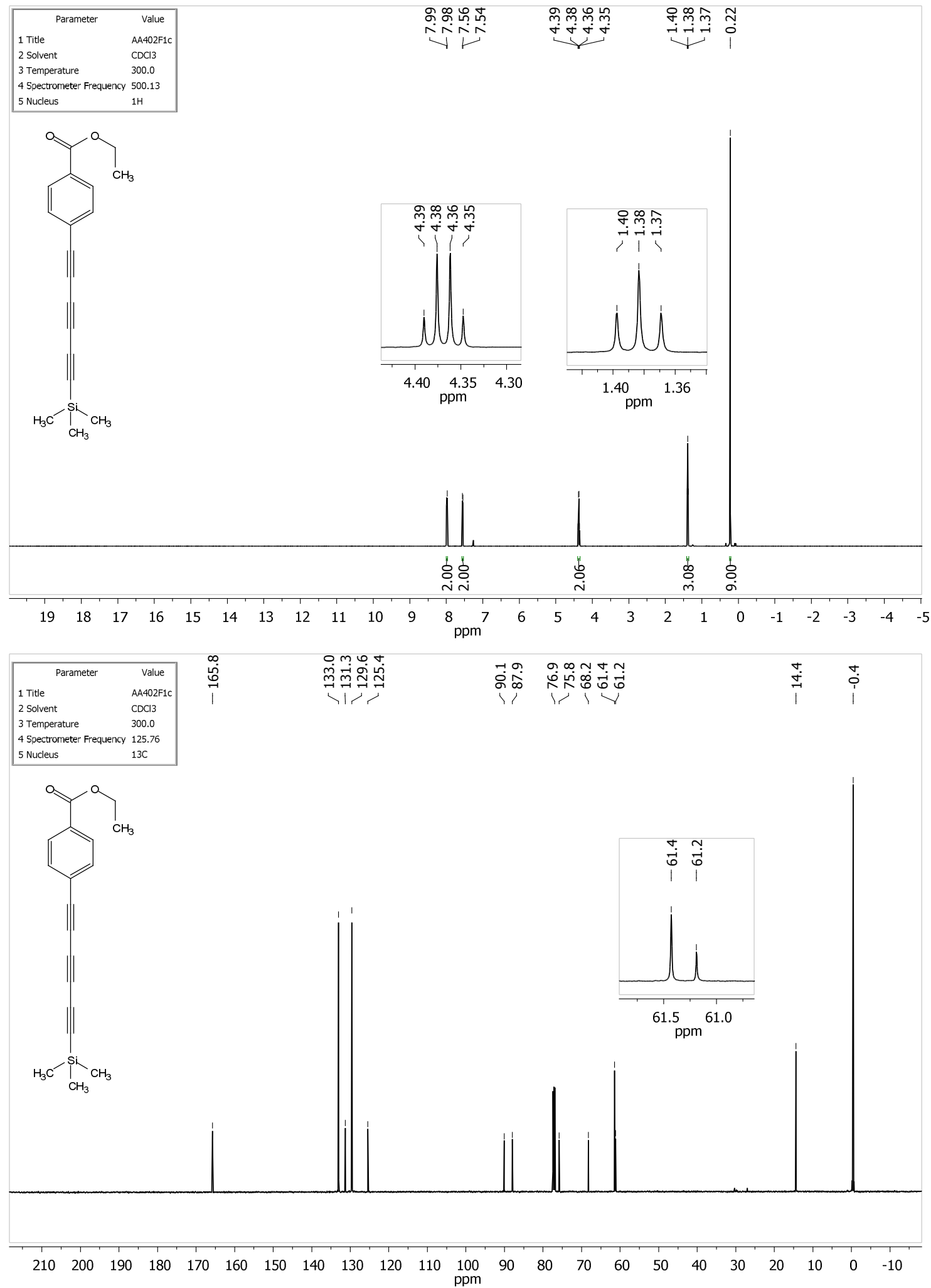


\section{(5-C6 $T$ TMS) 4-((Trimethylsilyl)hexatriynyl)phenyl acetate}
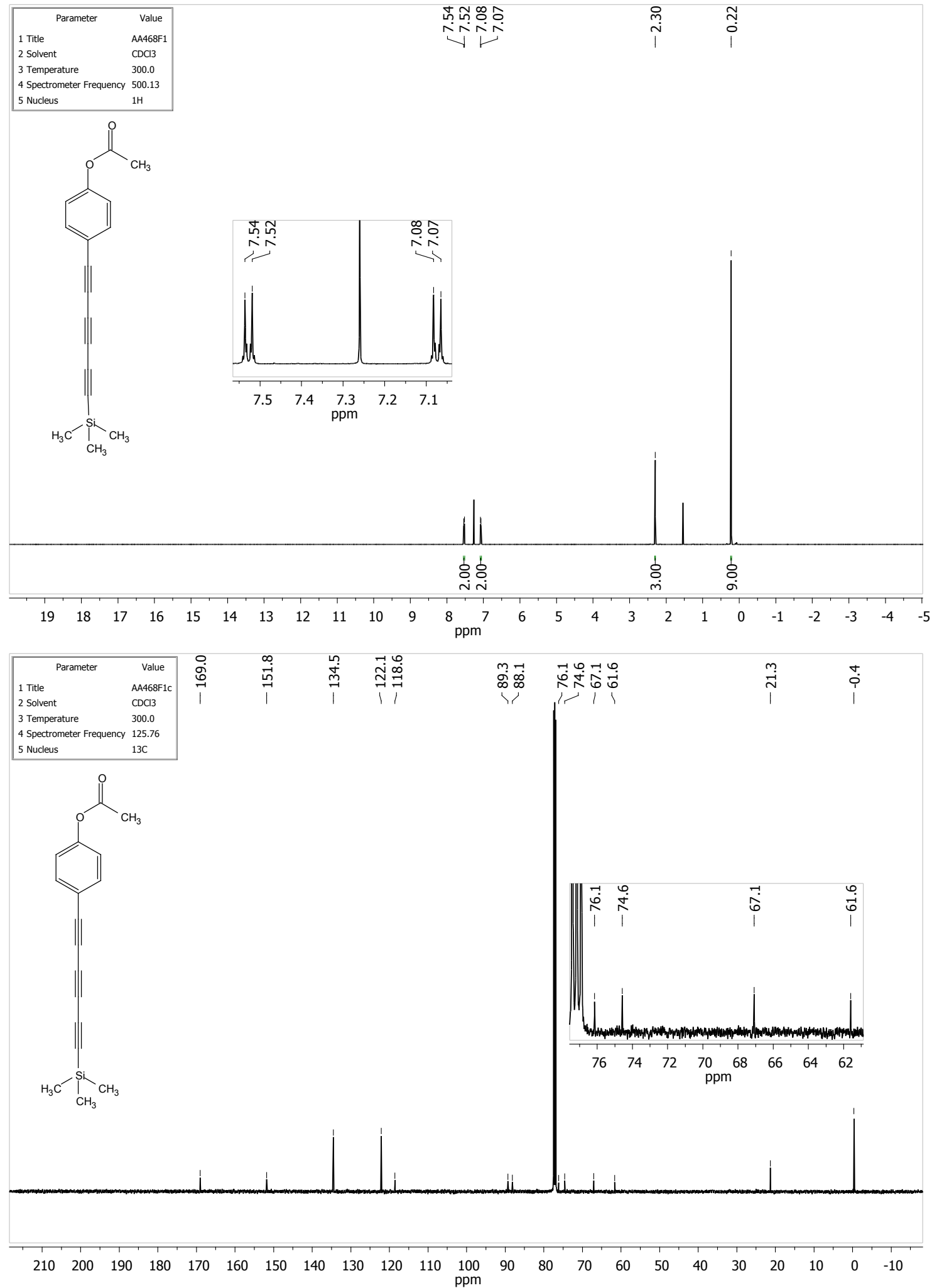


\section{(2- $\left.\mathrm{C}_{6} \mathrm{Br}\right)$ 4-(Bromohexatriynyl)nitrobenzene}
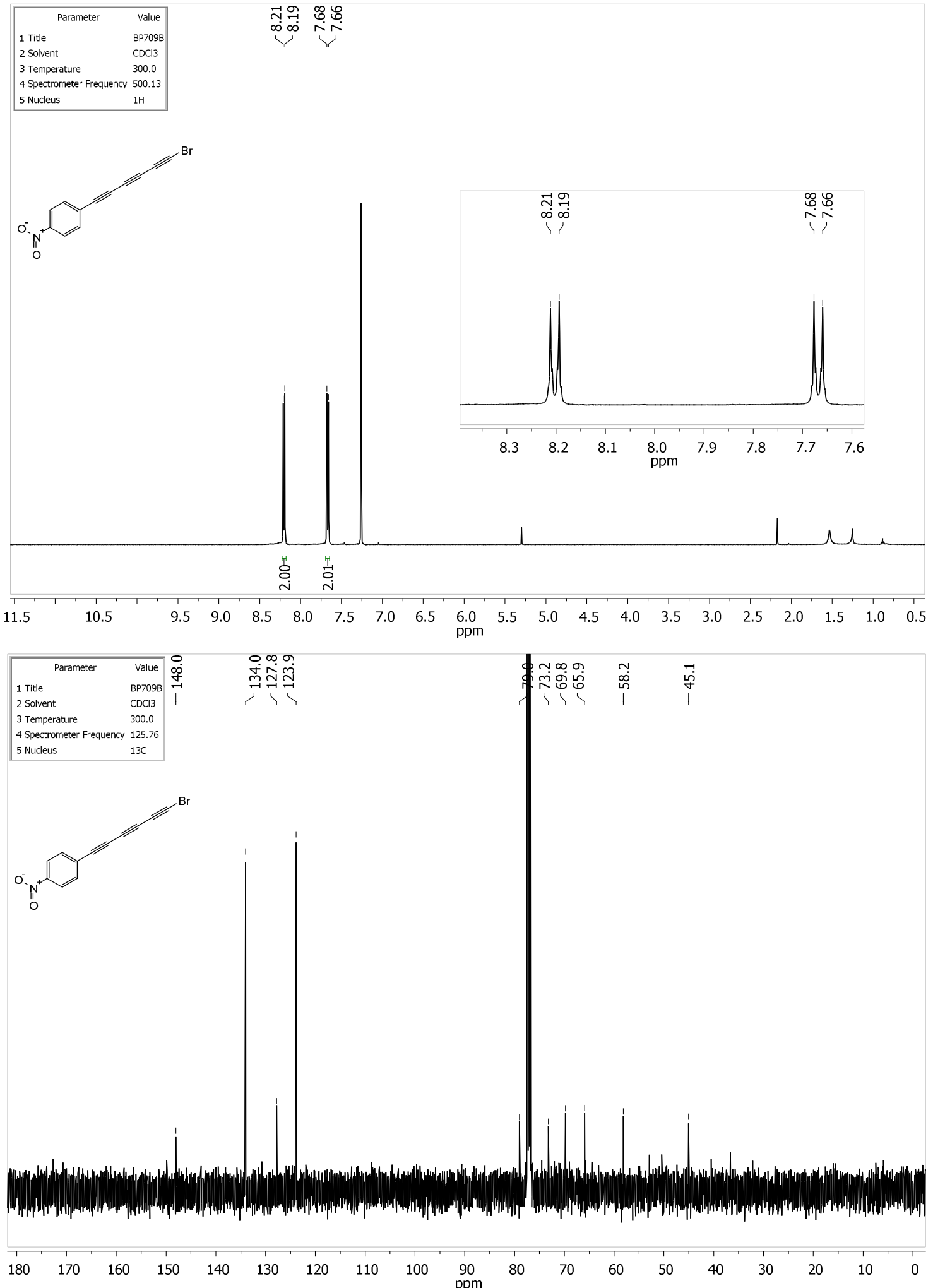


\section{(3- $\left.\mathrm{C}_{6} \mathrm{Br}\right)$ 1-(4-(Bromohexatriynyl)phenyl)ethanone}
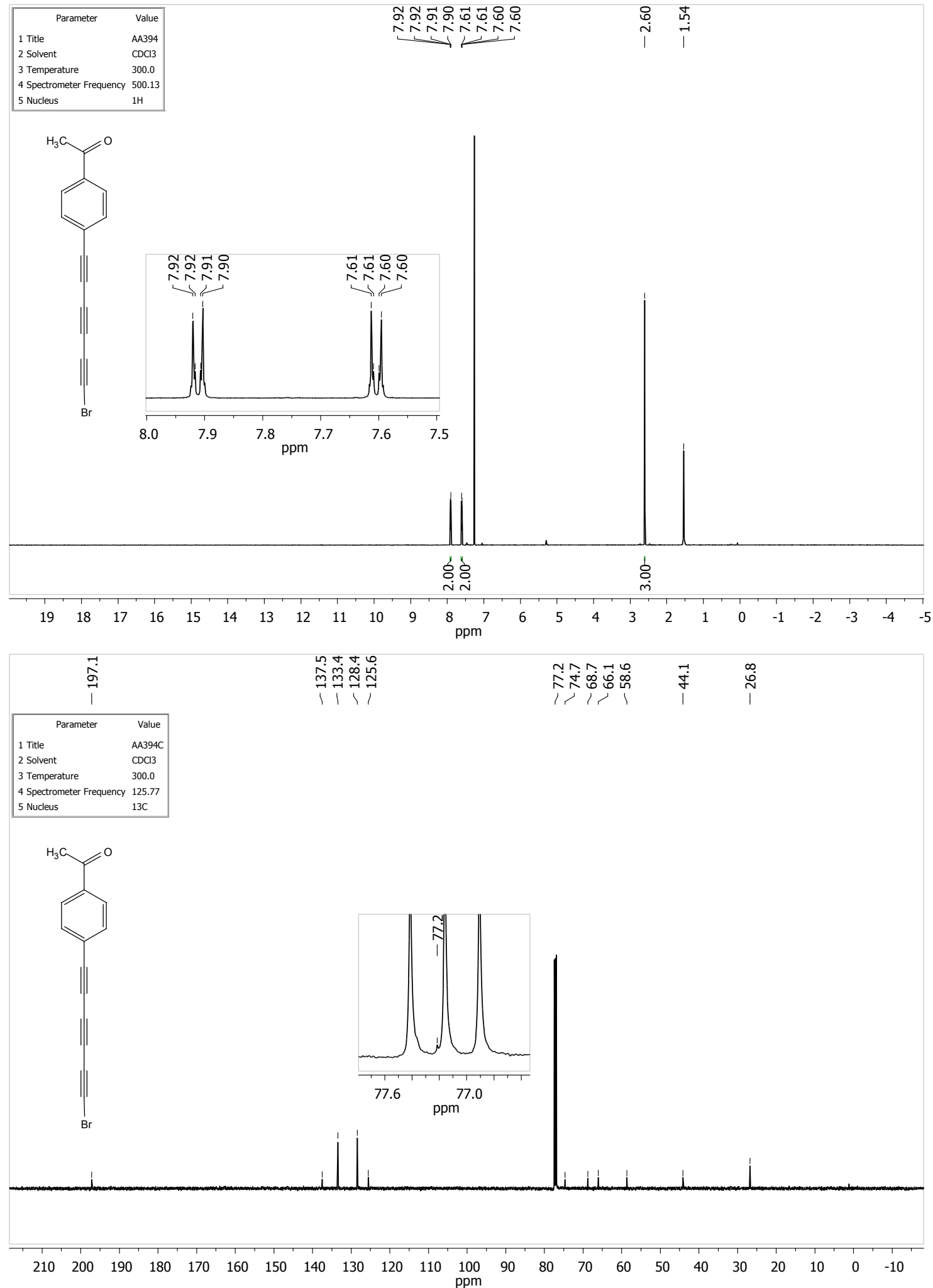


\section{(4- $\mathrm{C}_{6} \mathrm{Br}$ ) Ethyl 4-(bromohexatriynyl)benzoate}
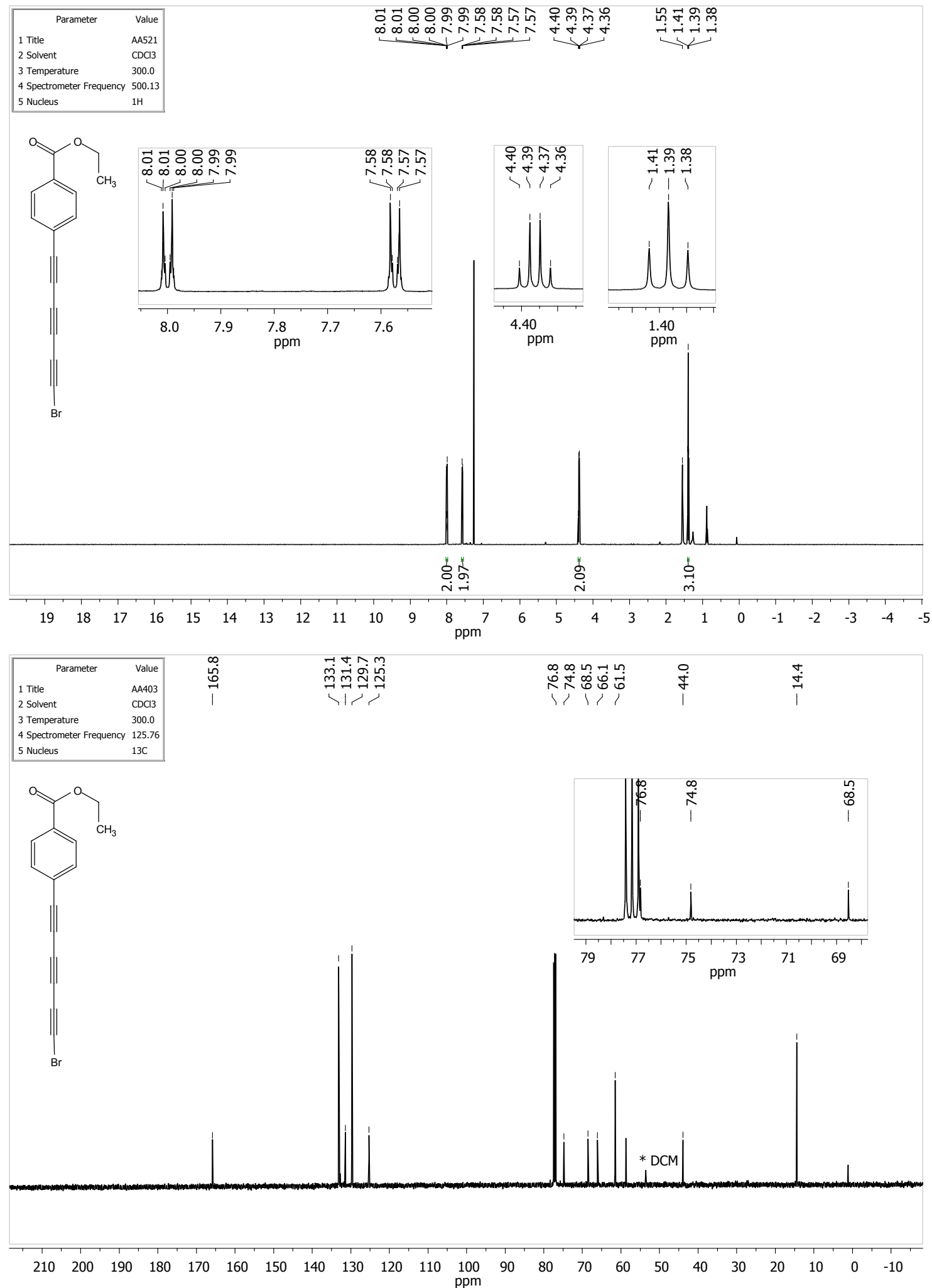


\section{(5- $\left.\mathrm{C}_{6} \mathrm{Br}\right)$ 4-(Bromohexatriynyl)phenyl acetate}
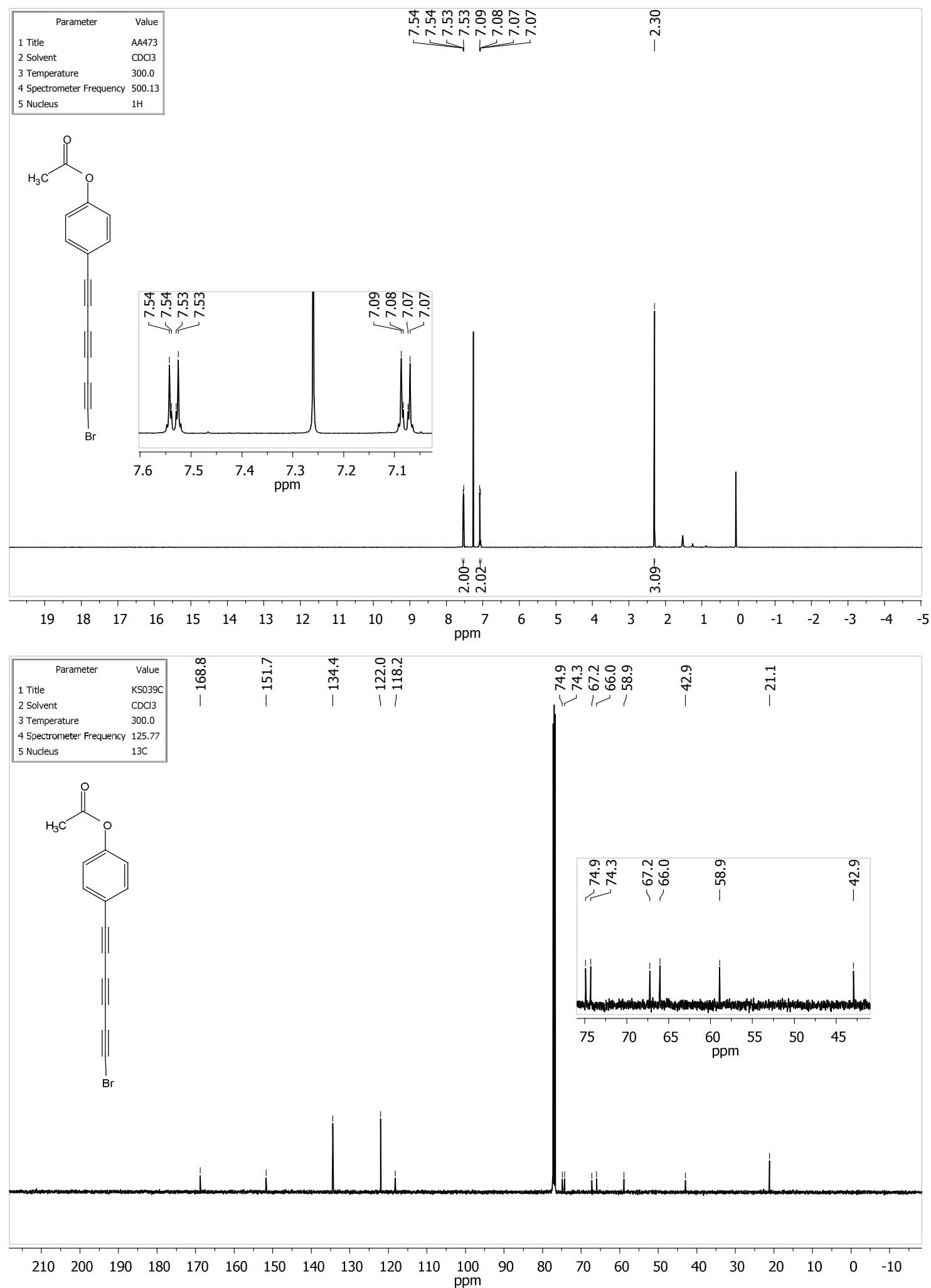


\section{(1-C 6 THI-H) 4-((4,5,6,7-Tetrahydro-1H-indol-2-yl)hexatriynyl)benzonitrile}
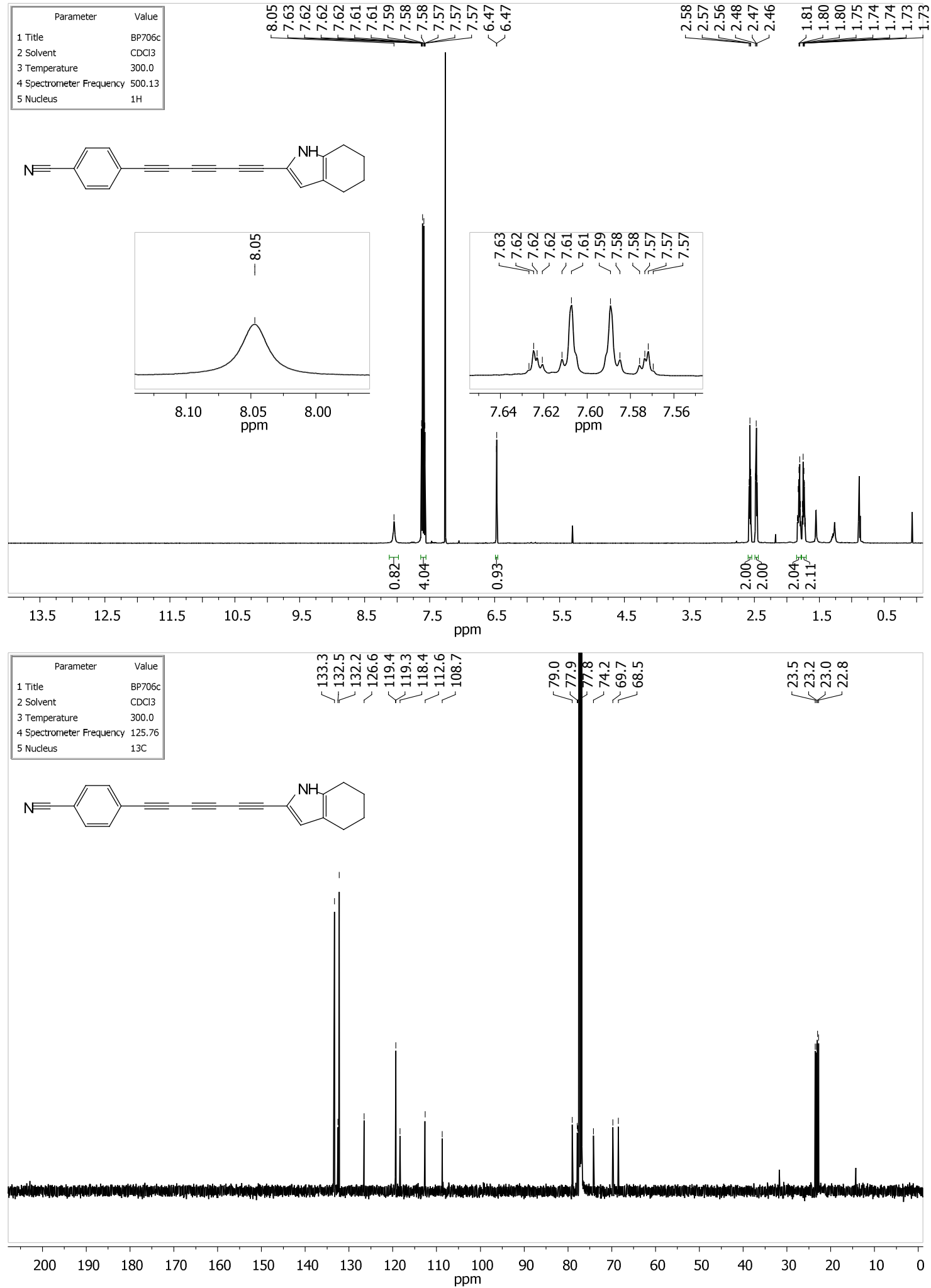
(1-C 6 THI-Me) 4-((1-Methyl-4,5,6,7-tetrahydro-1H-indol-2-yl)hexatriynyl)benzonitrile
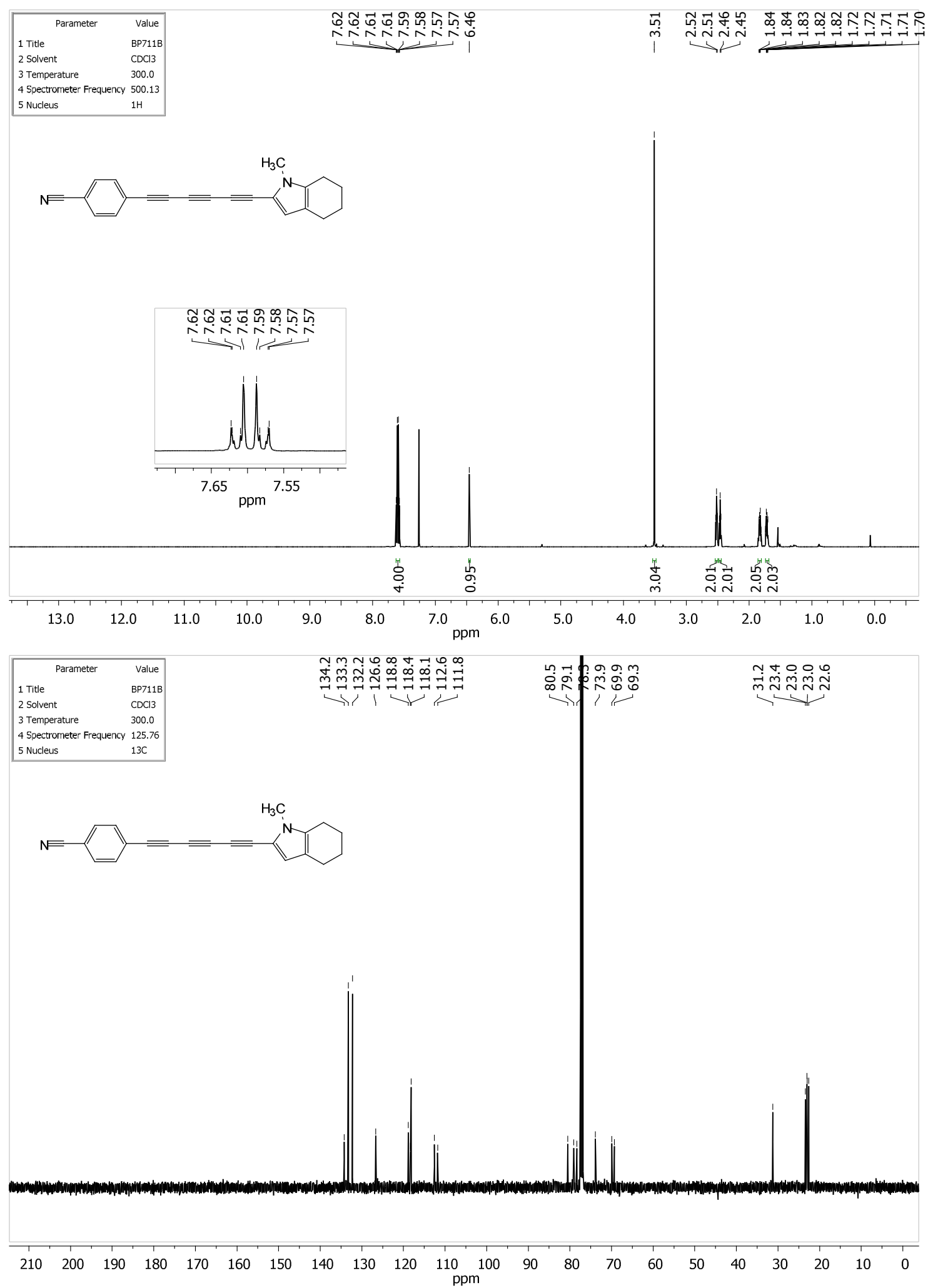
(1-C 6 THI-Vin) 4-((1-Vinyl-4,5,6,7-tetrahydro-1H-indol-2-yl)hexatriynyl)benzonitrile
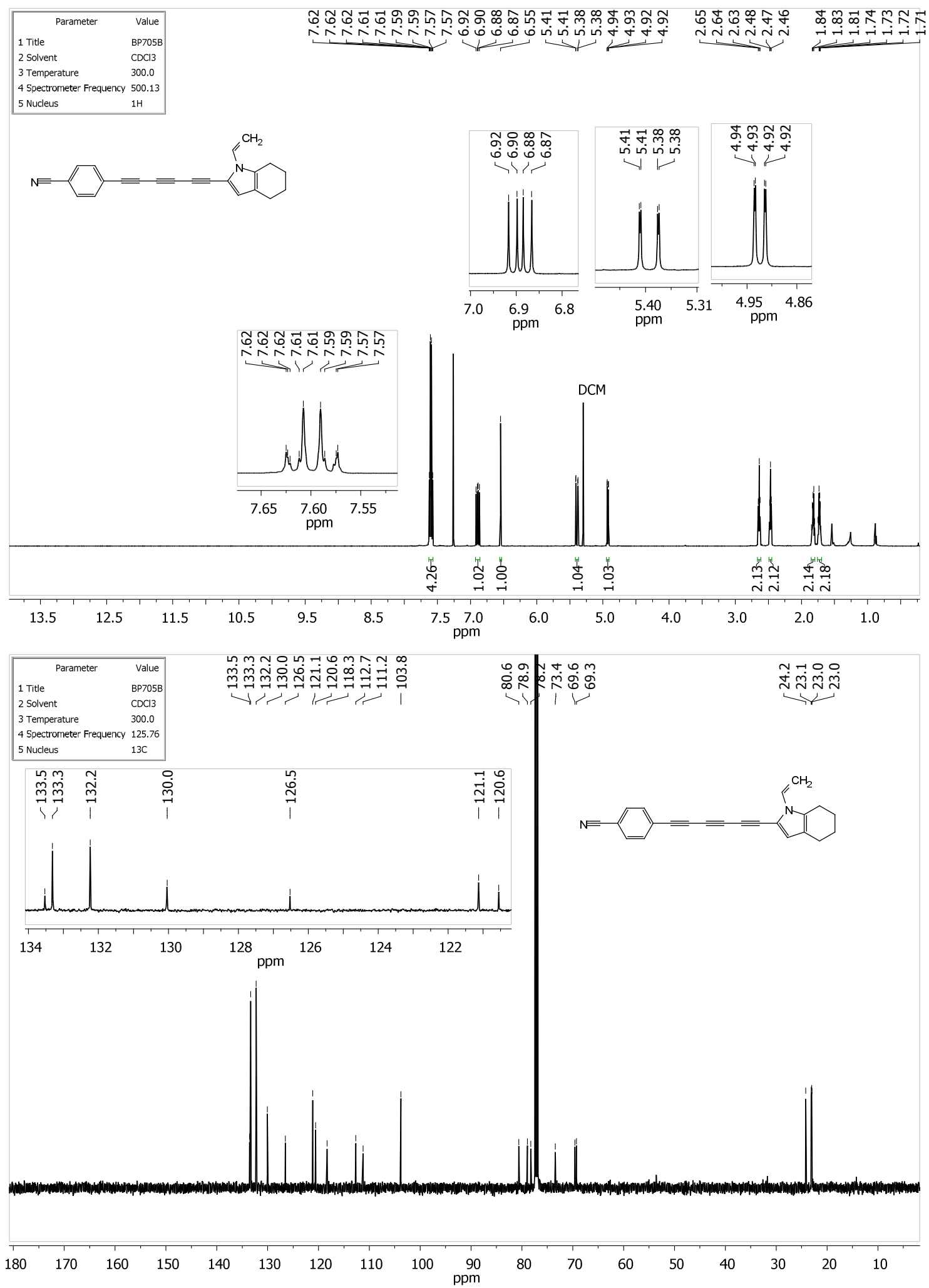
(1-C 6 THI-Bn) 4-((1-Benzyl-4,5,6,7-tetrahydro-1H-indol-2-yl)hexatriynyl)benzonitrile
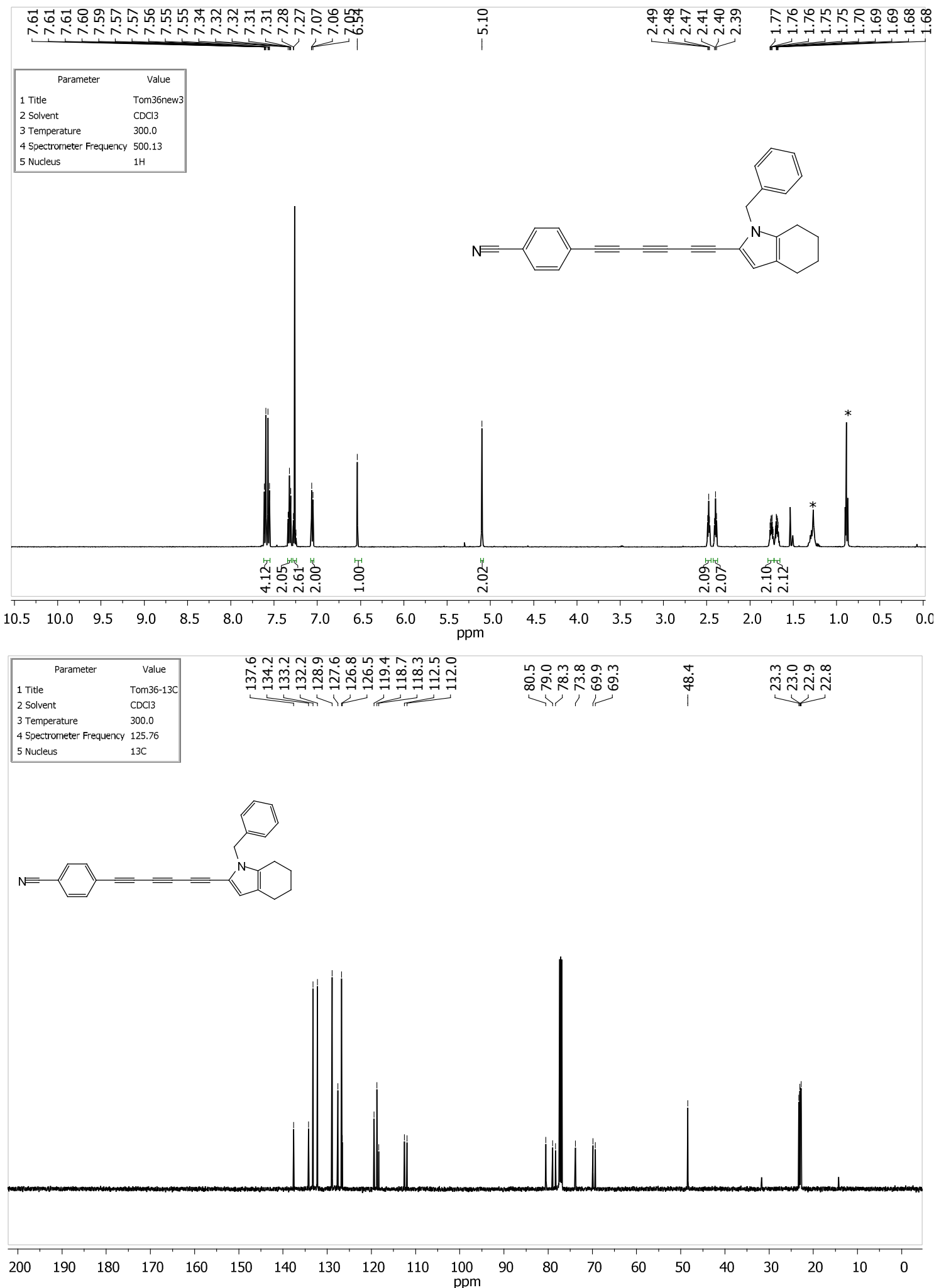
(2-C 6 THI-H) 2-((4-Nitrophenyl)hexatriynyl)-4,5,6,7-tetrahydro-1H-indole
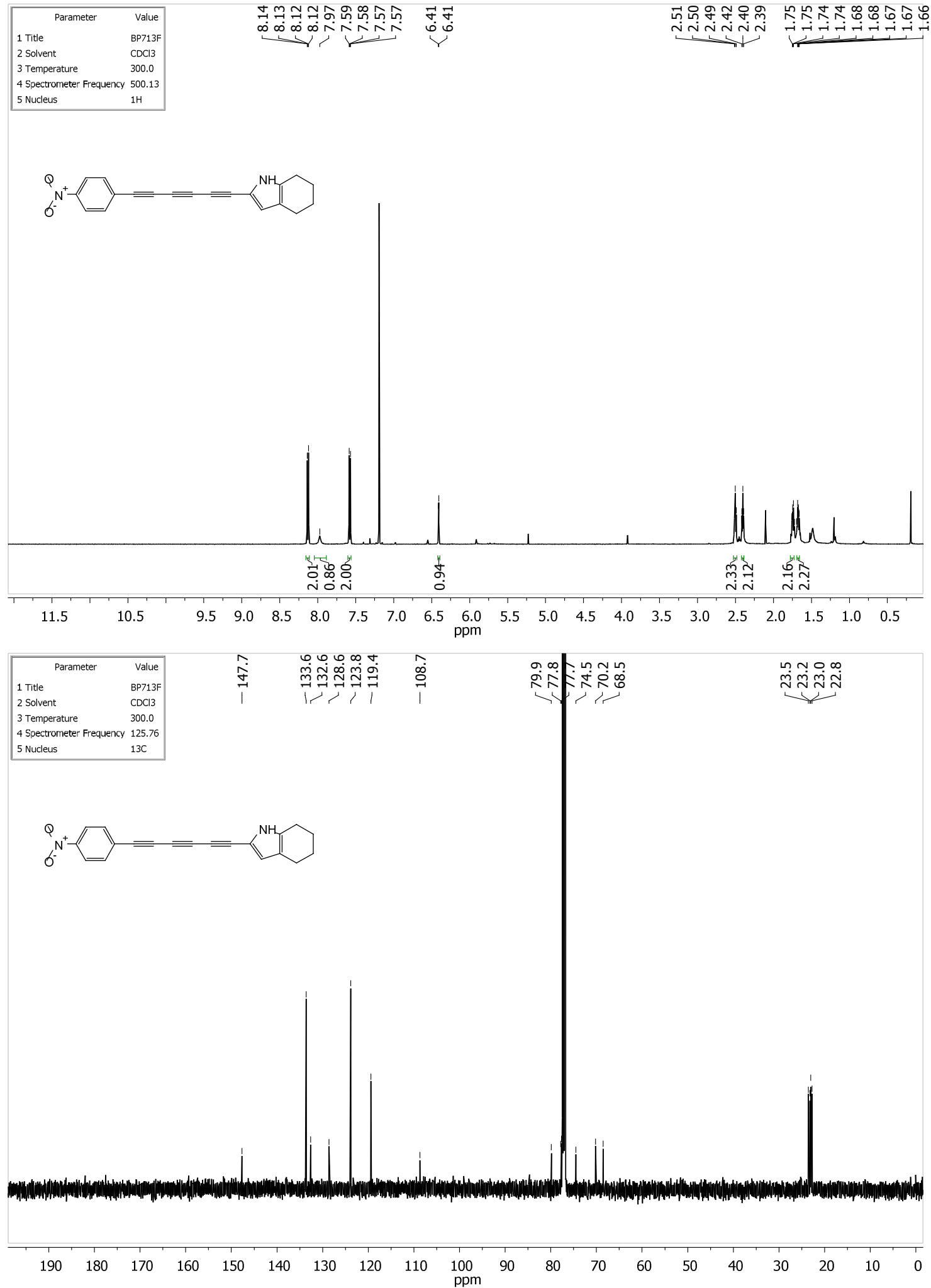
(2-C 6 THI-Me) 1-Methyl-2-((4-nitrophenyl)hexatriynyl)-4,5,6,7-tetrahydro-1 $H$-indole
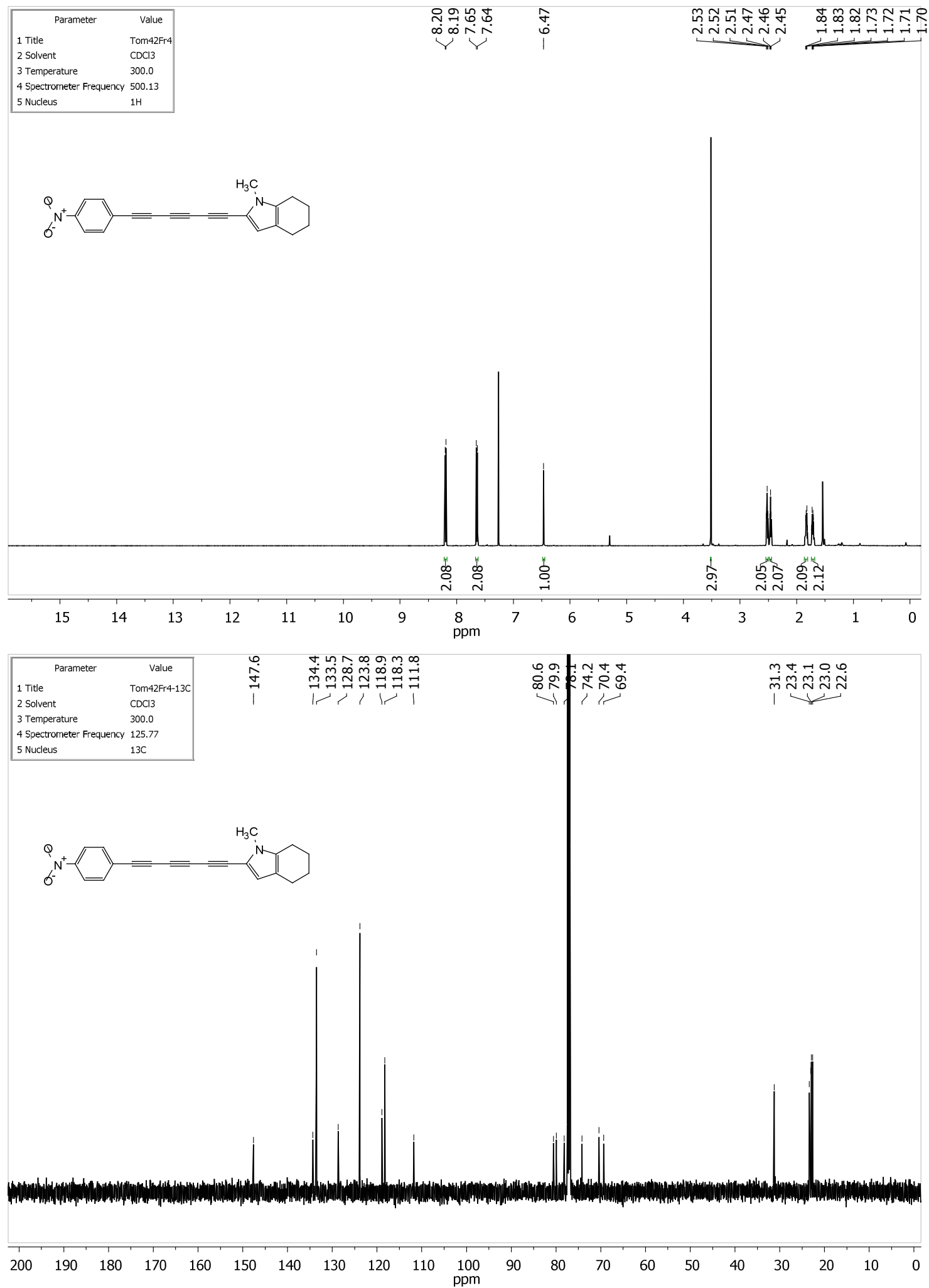
(2-C 6 THI-Vin) 1-Vinyl-2-((4-nitrophenyl)hexatriynyl)-4,5,6,7-tetrahydro-1H-indole
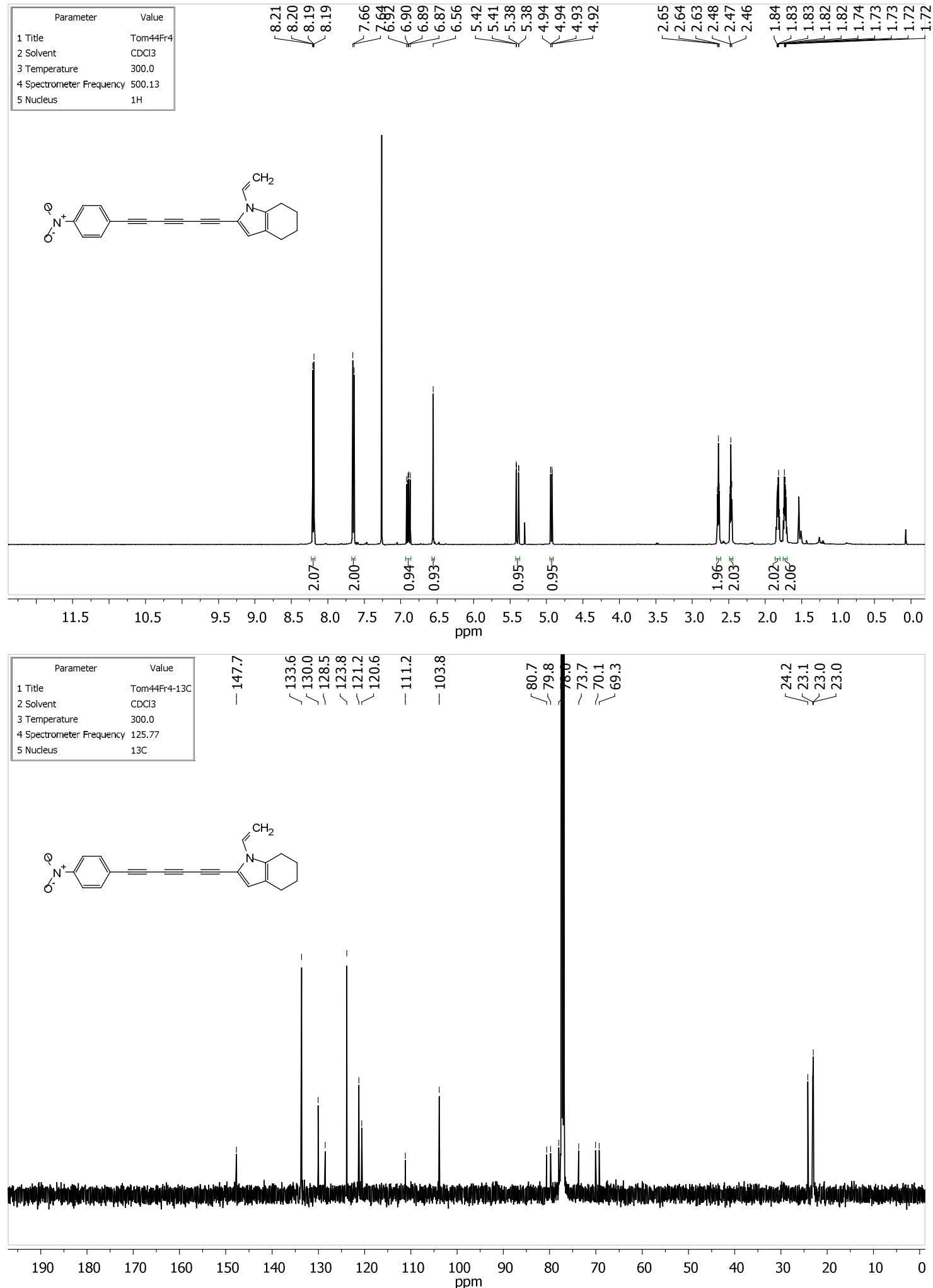
(2-C 6 THI-Bn) 1-Benzyl-2-((4-nitrophenyl)hexatriynyl)-4,5,6,7-tetrahydro-1H-indole
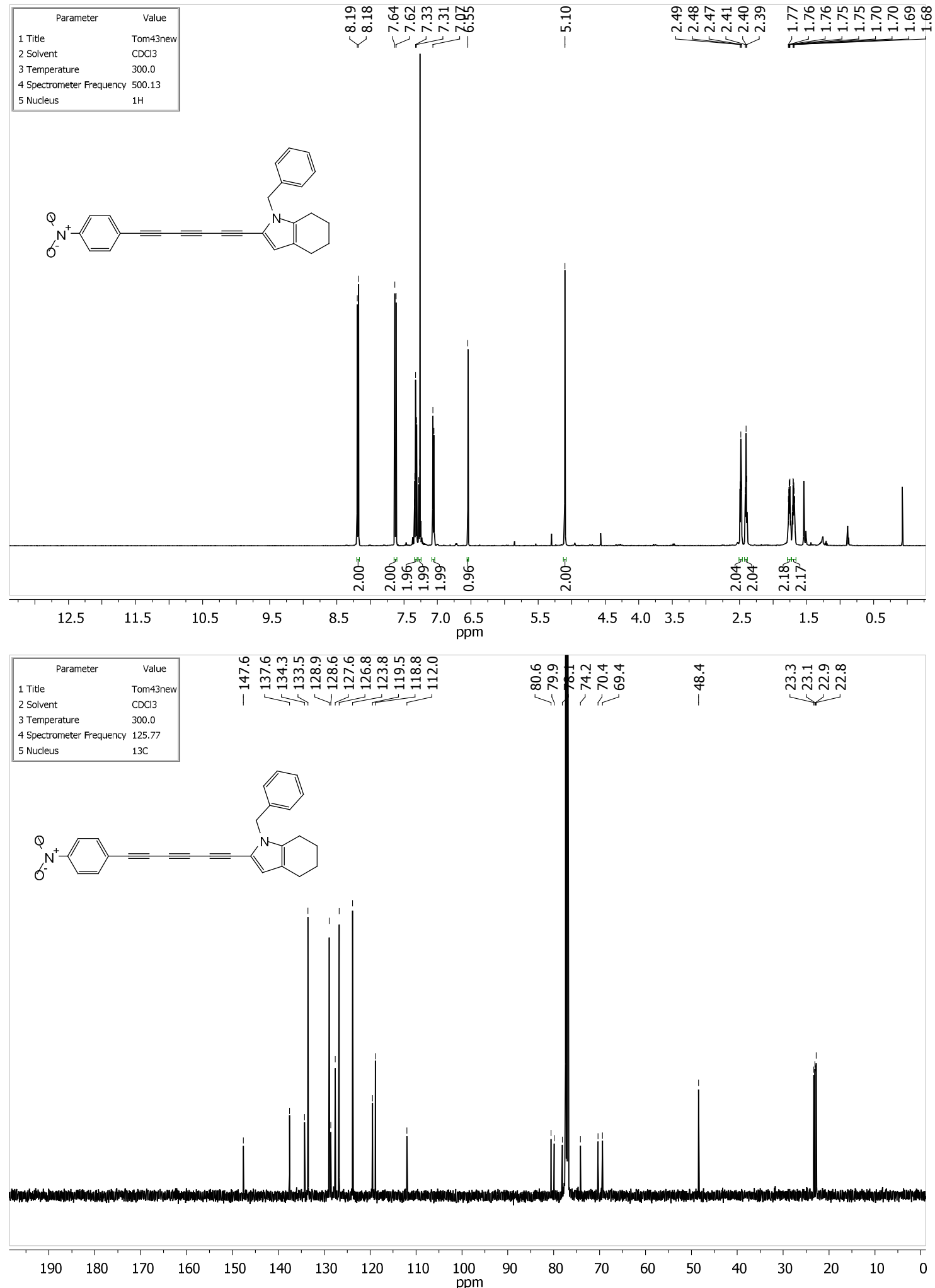
(3-C 6 THI-H), 1-(4-((4,5,6,7-Tetrahydro-1H-indol-2-yl)hexatriynyl)phenyl)ethanone
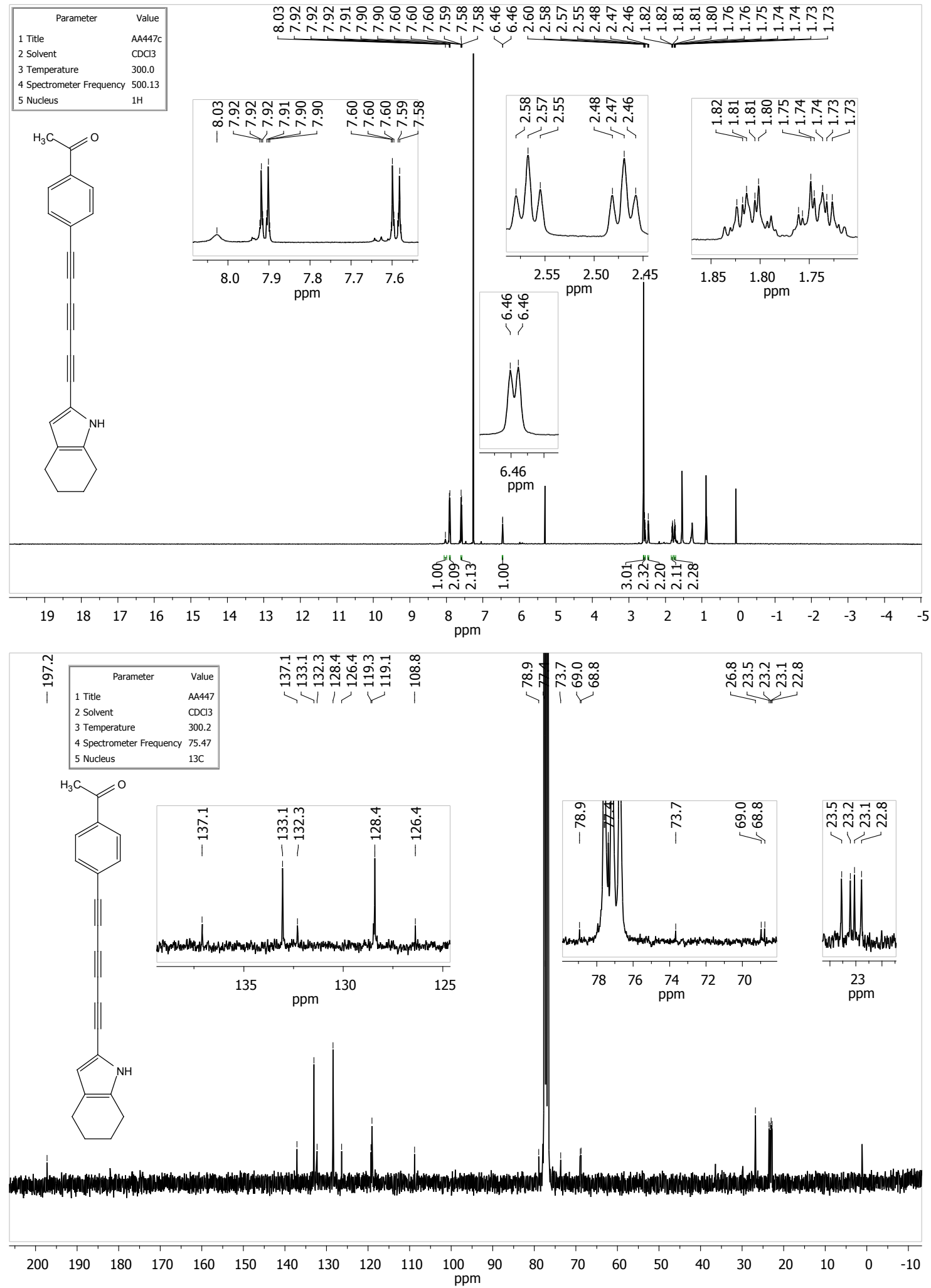


\section{(3-C 6 THI-Me),}

\section{1-(4-((1-Methyl-4,5,6,7-tetrahydro-1H-indol-2-yl)hexatriynyl)phenyl)ethanone}
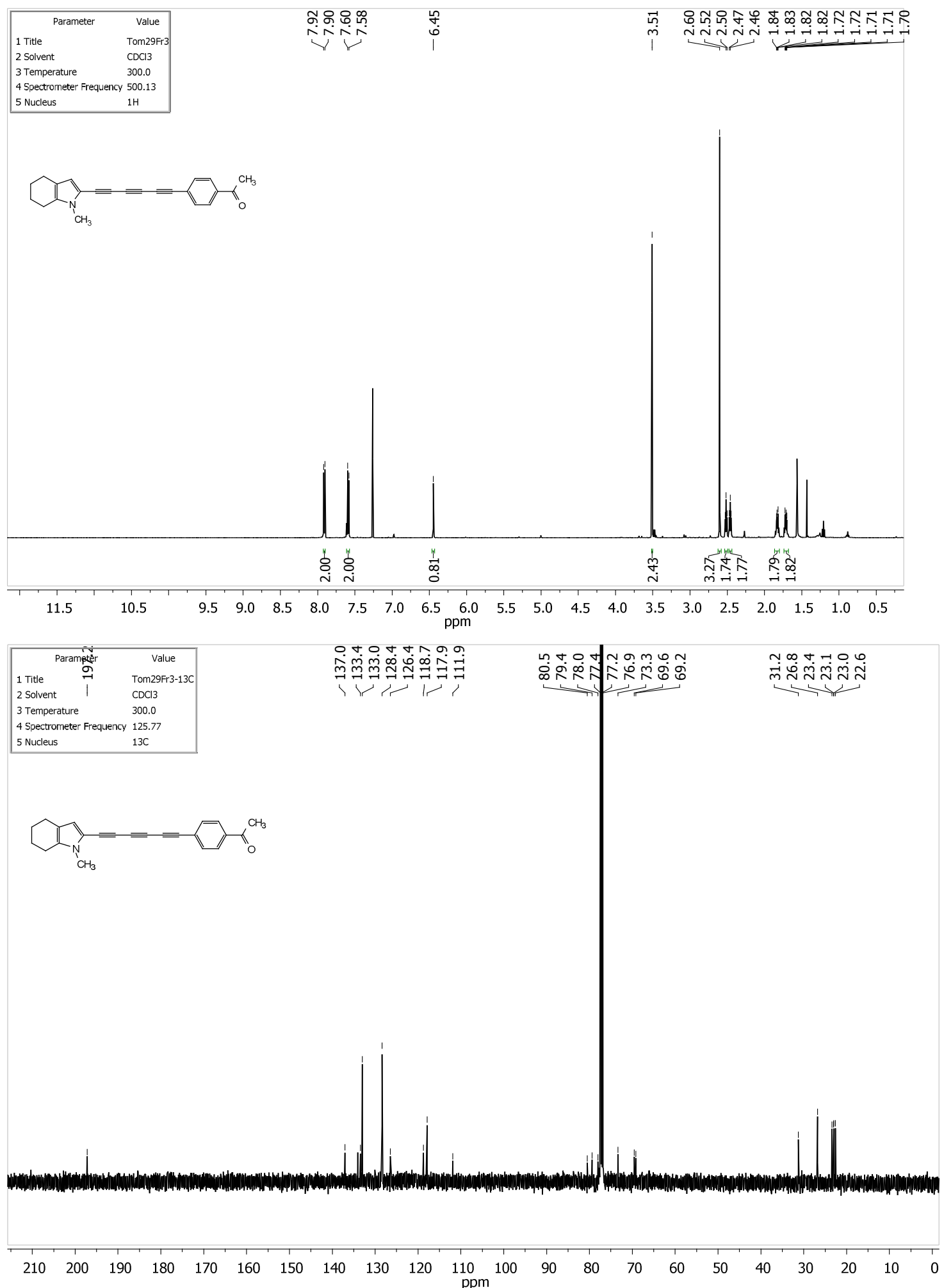


\section{(3-C 6 THI-Vin),}

\section{1-(4-((1-Vinyl-4,5,6,7-tetrahydro-1H-indol-2-yl)hexatriynyl)phenyl)ethanone}
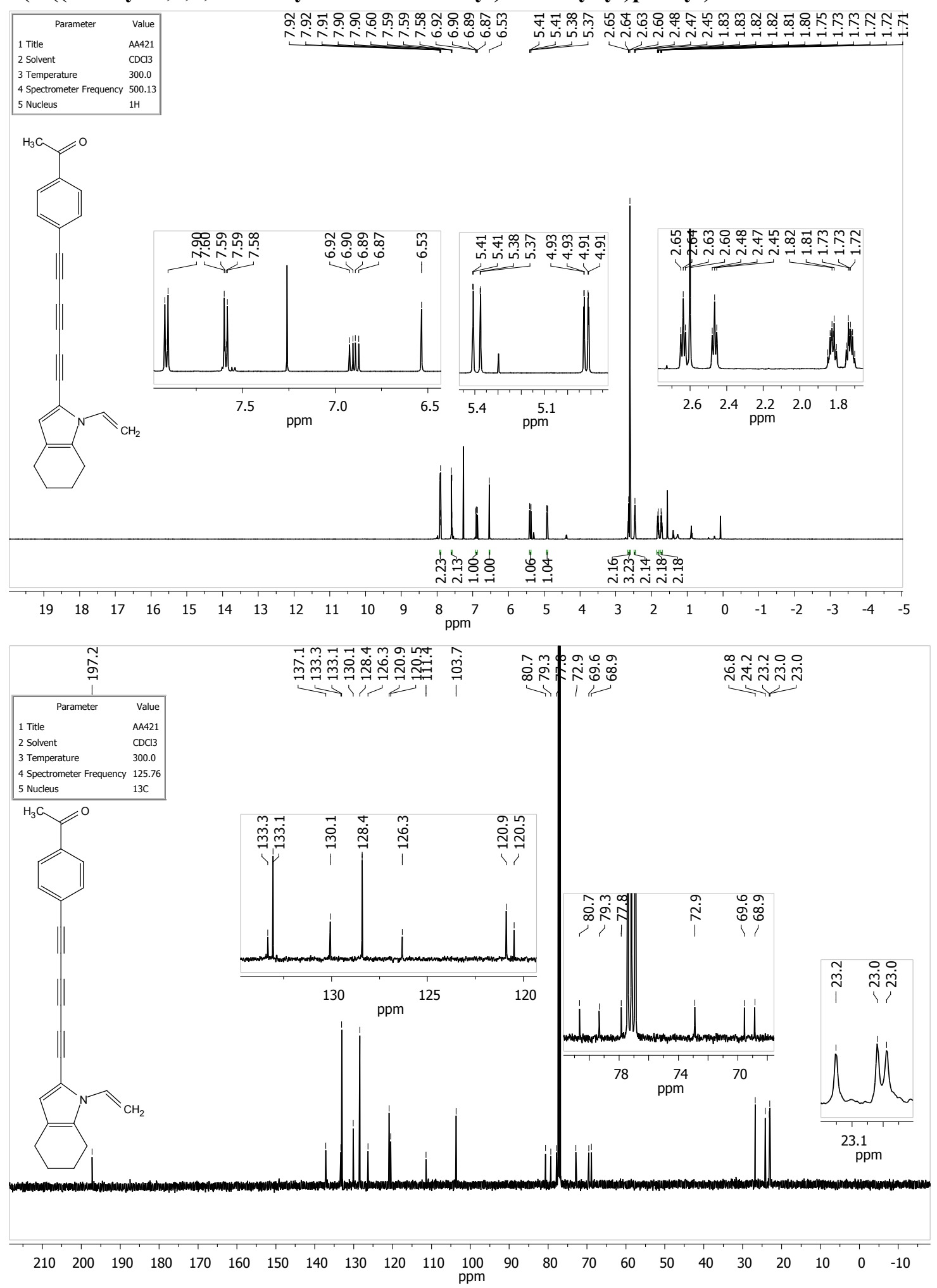


\section{(3-C 6 THI-Bn),}

\section{1-(4-((1-Benzyl-4,5,6,7-tetrahydro-1H-indol-2-yl)hexatriyn-yl)phenyl)ethanon}
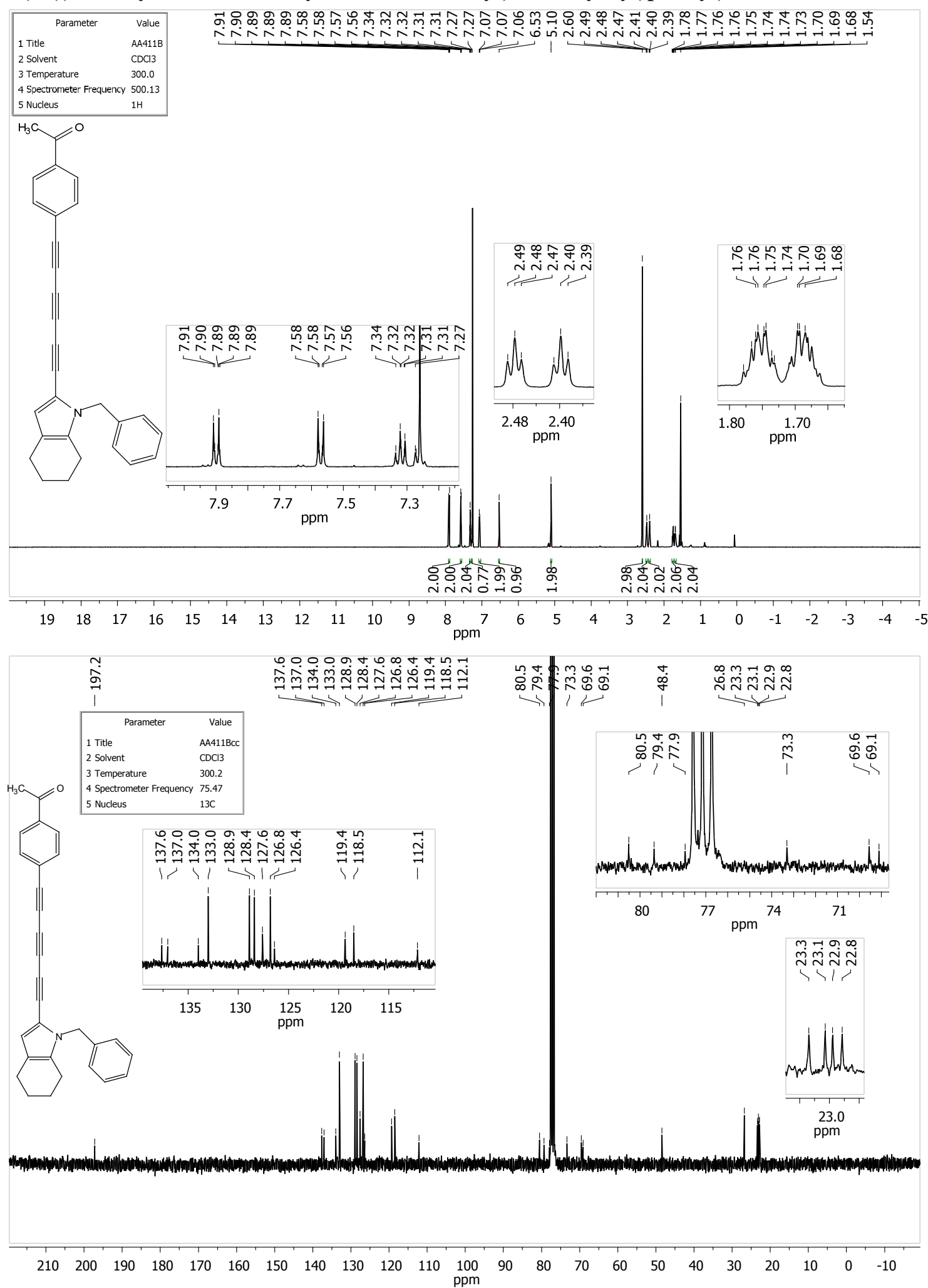
(4-C 6 THI-H), Ethyl 4-((4,5,6,7-tetrahydro-1H-indol-2-yl)hexatriynyl)benzoate
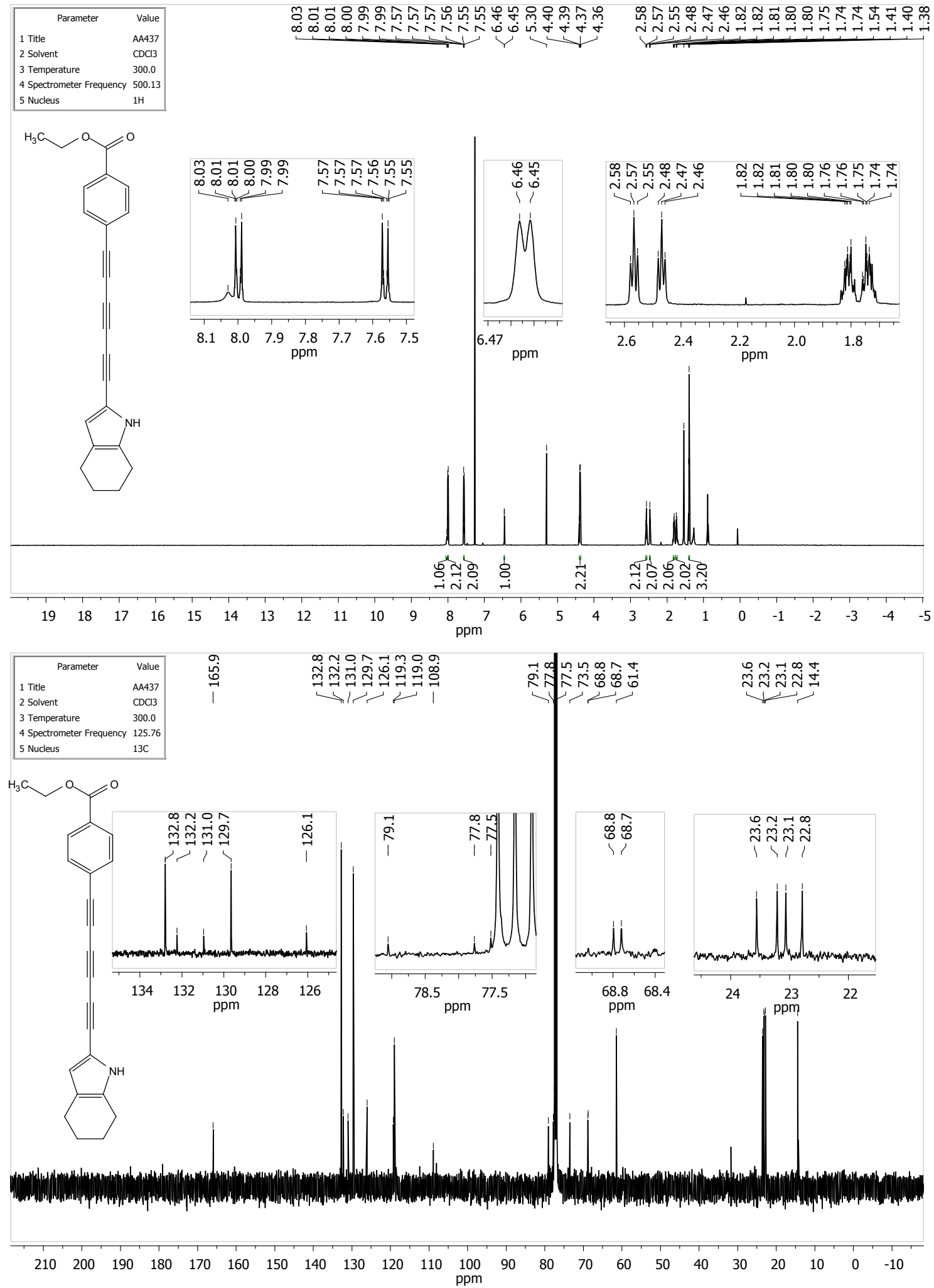


\section{(4-C 6 THI-Me),}

Ethyl 4-((1-methyl-4,5,6,7-tetrahydro-1H-indol-2-yl)hexatriynyl)benzoate
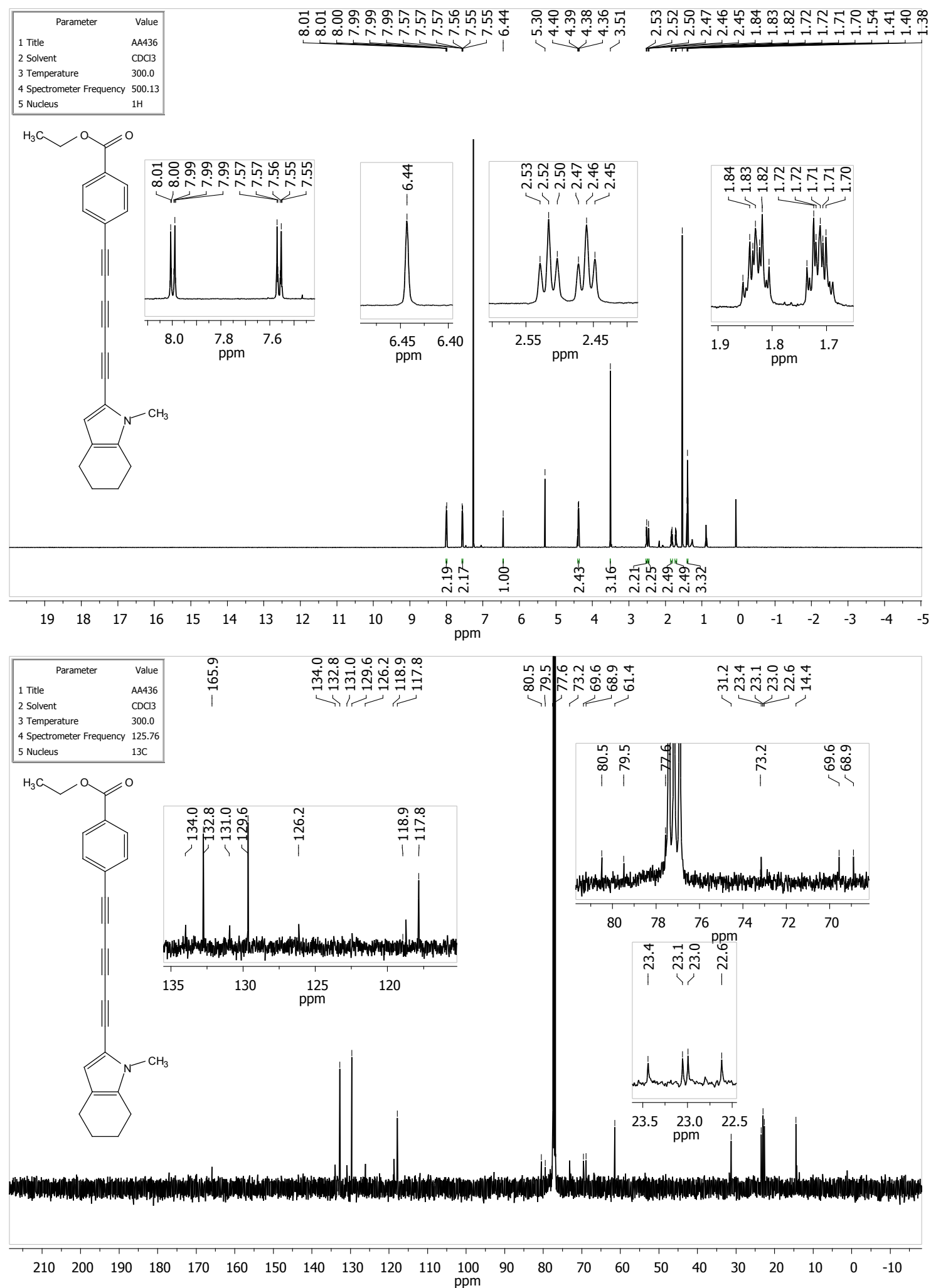
(4-C 6 THI-Vin), Ethyl 4-((1-vinyl-4,5,6,7-tetrahydro-1H-indol-2-yl)hexatriynyl)benzoate
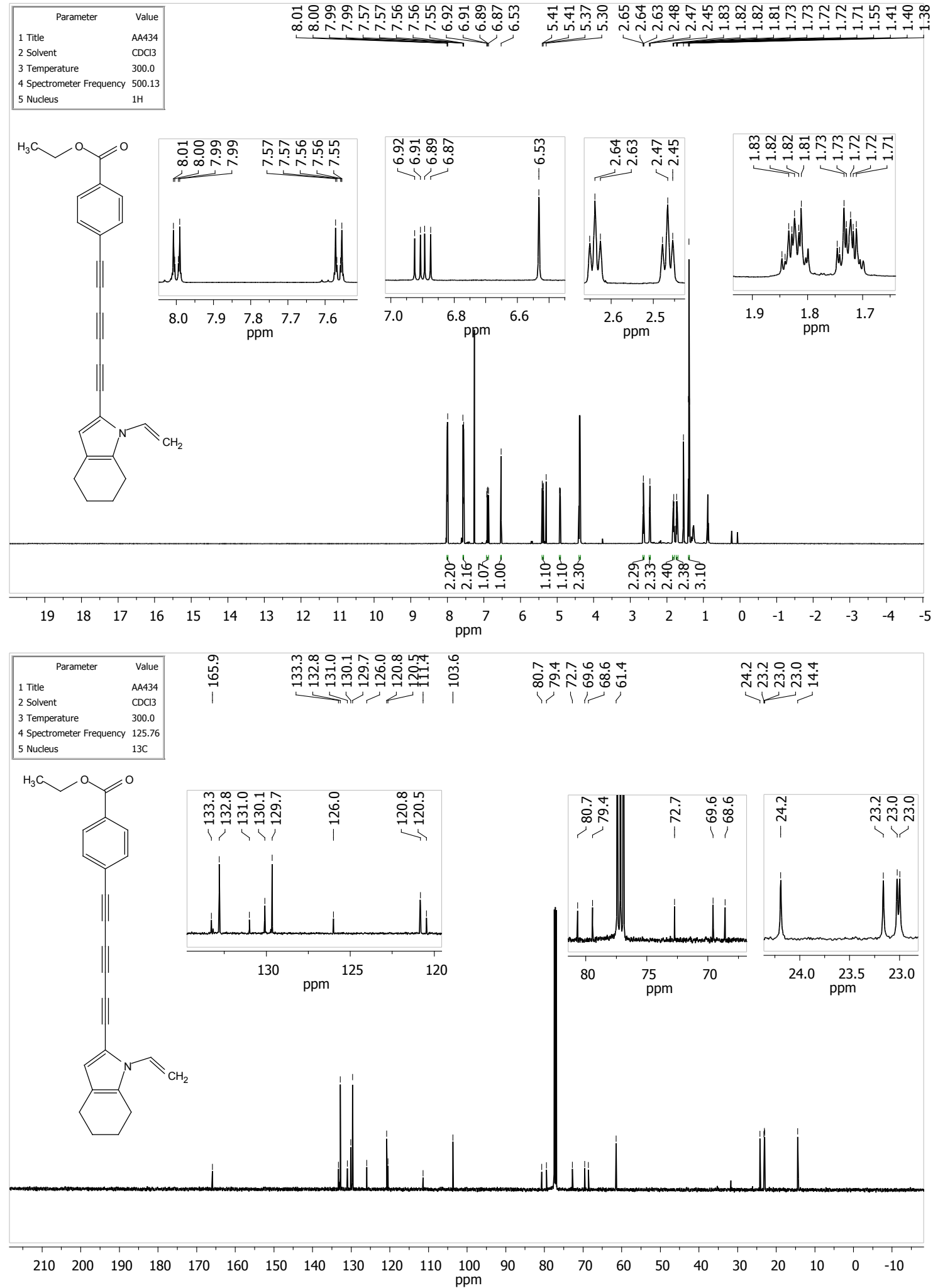
(4-C 6 THI-Bn), Ethyl 4-((1-benzyl-4,5,6,7-tetrahydro-1H-indol-2-yl)hexatriynyl)benzoate
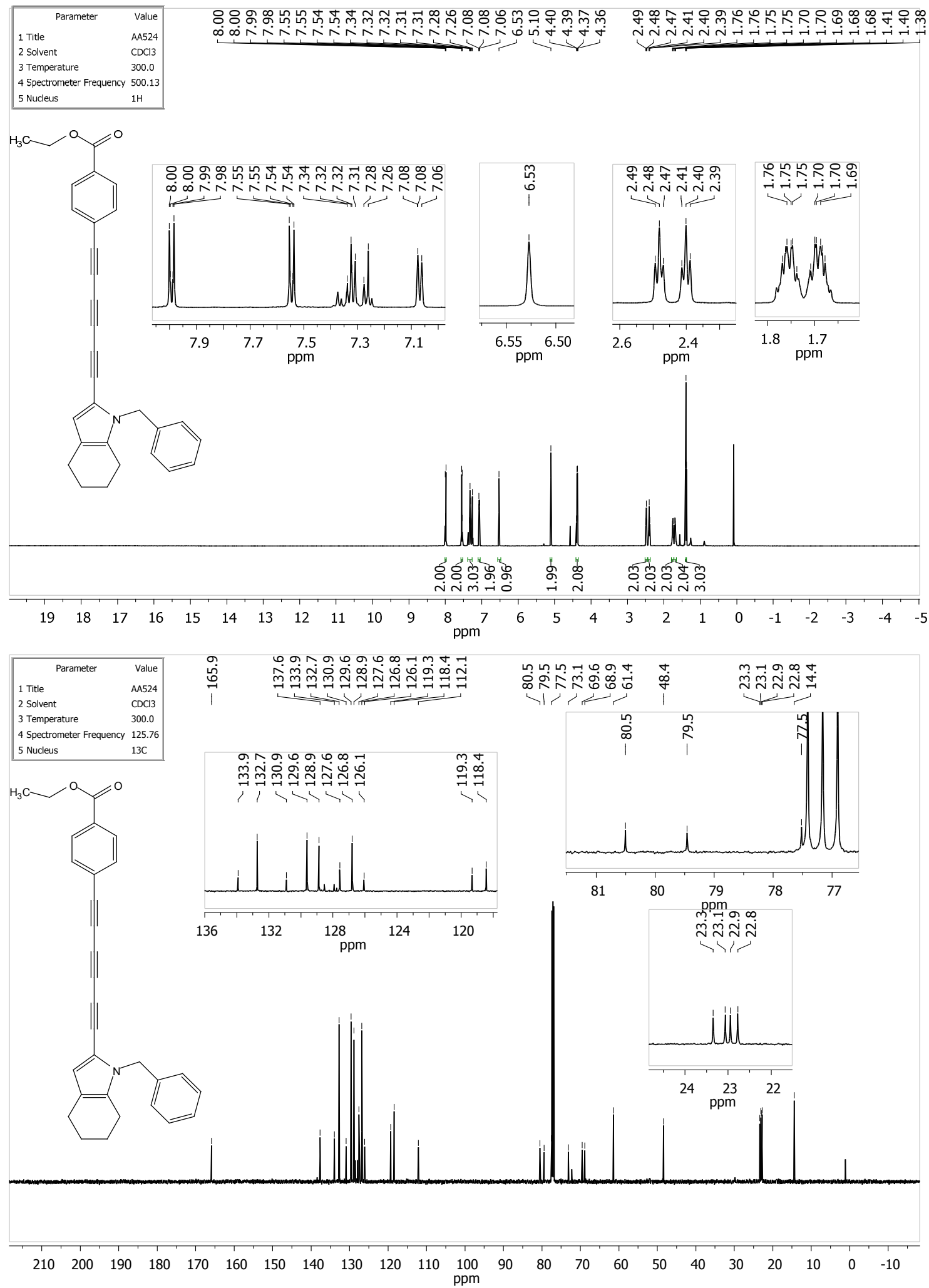
(5-C 6 THI-Me), 4-((1-Methyl-4,5,6,7-tetrahydro-1H-indol-2-yl)hexatriynyl)phenyl acetate
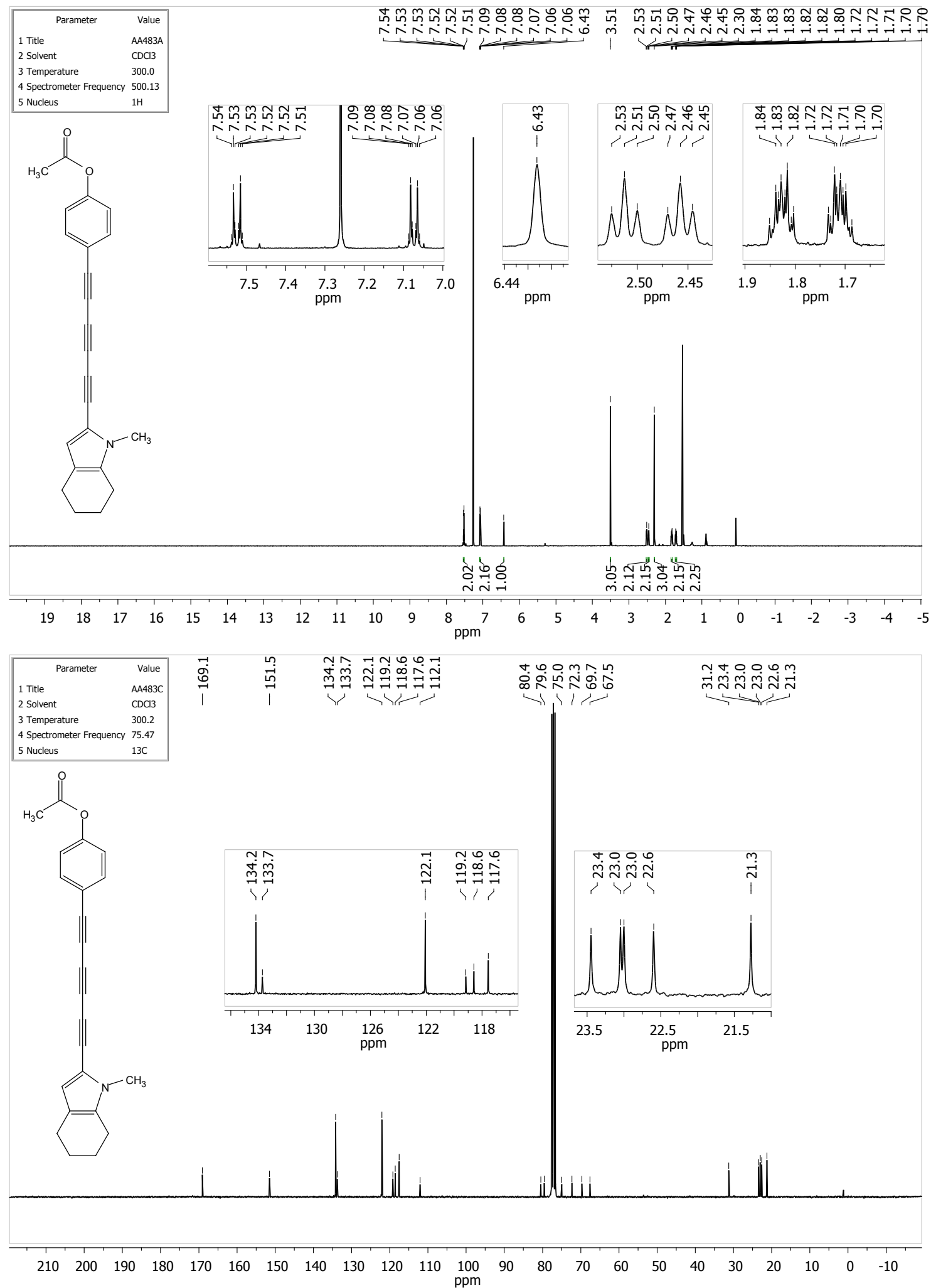
(5-C 6 THI-Vin), 4-((1-Vinyl-4,5,6,7-tetrahydro-1H-indol-2-yl)hexatriynyl)phenyl acetate
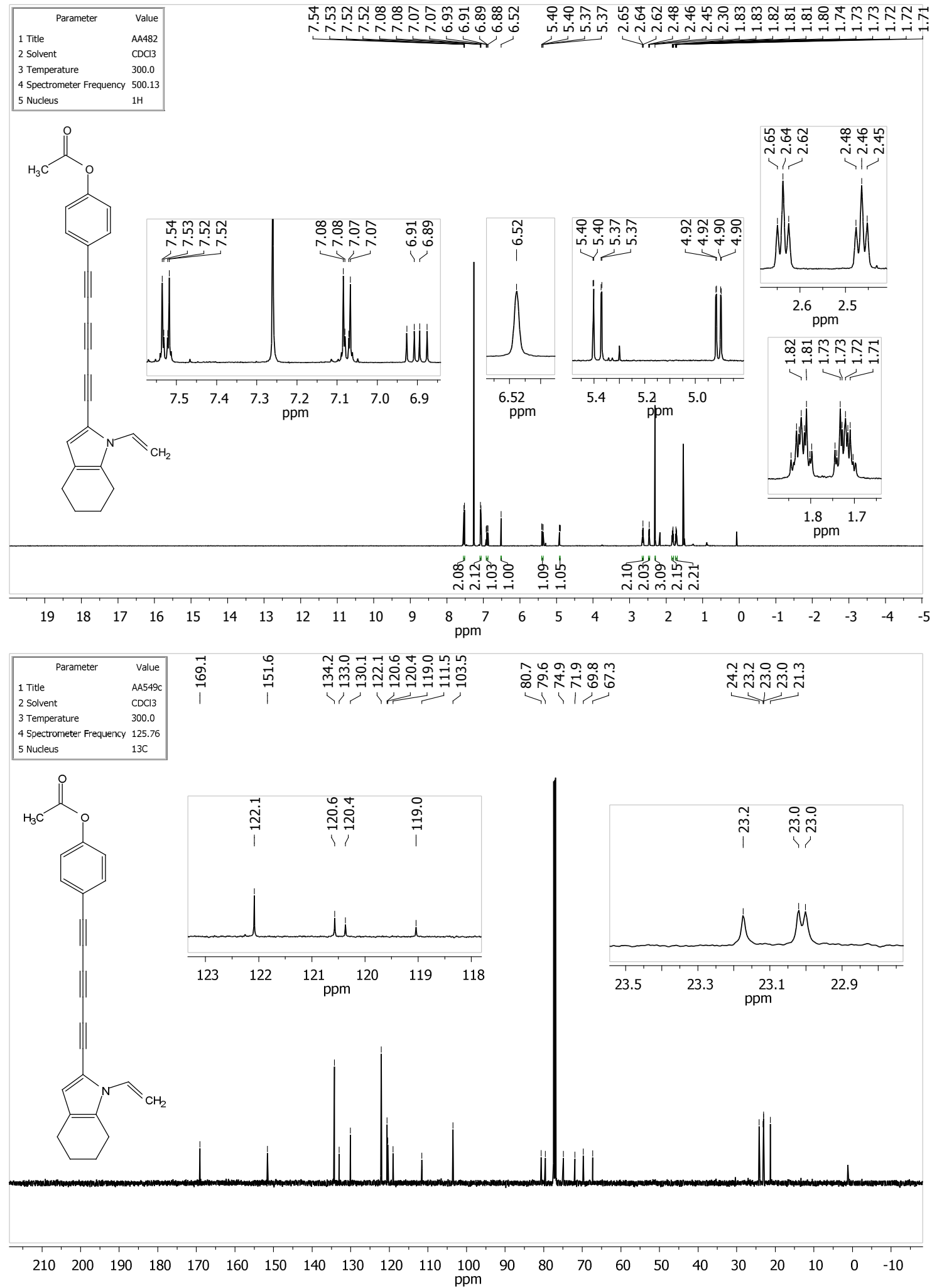
(5-C 6 THI-Bn), 4-((1-Benzyl-4,5,6,7-tetrahydro-1H-indol-2-yl)hexatriynyl)phenyl acetate
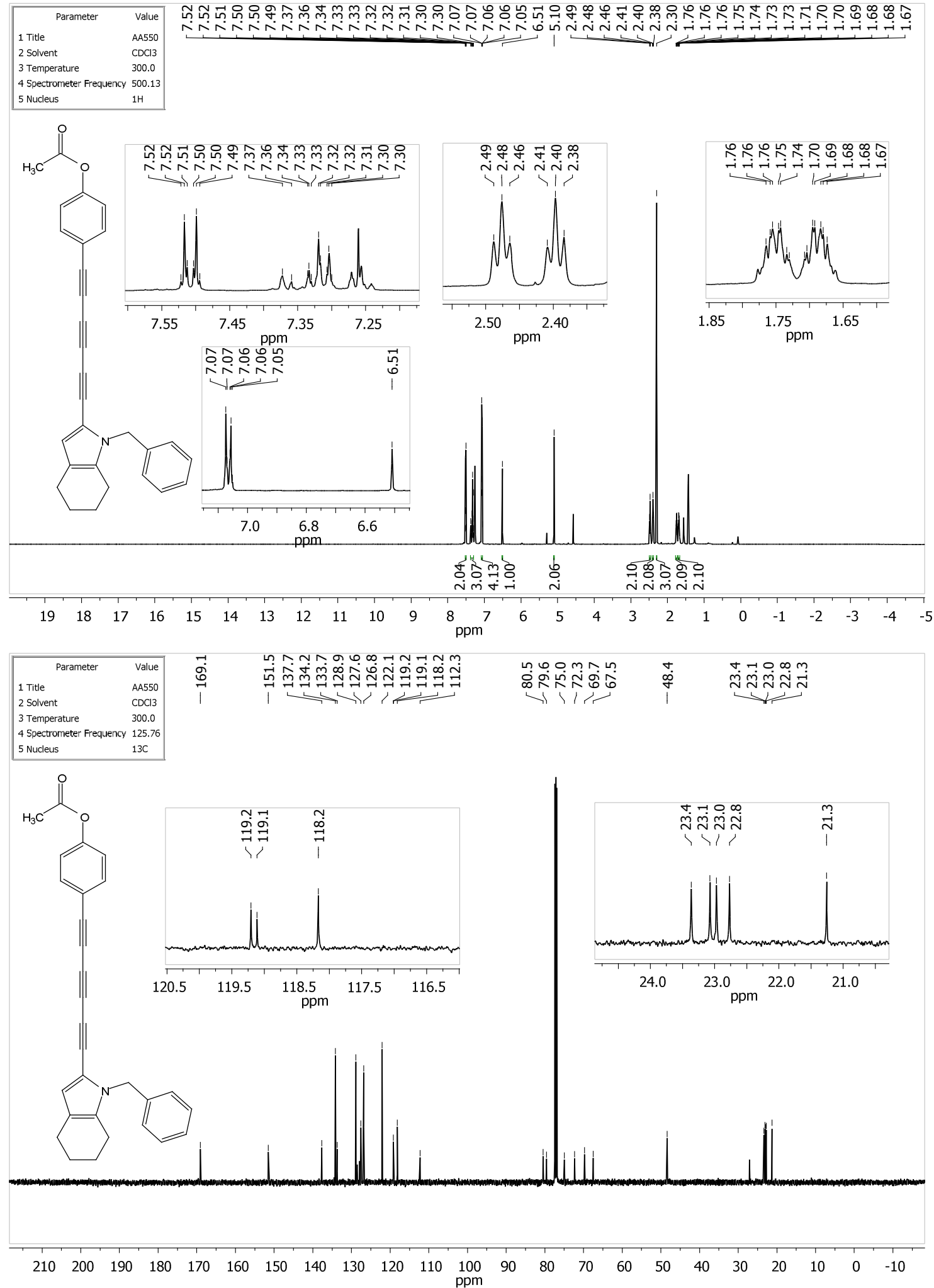
(5-C 6 THI-H), 4-((4,5,6,7-Tetrahydro-1H-indol-2-yl)hexatriynyl)phenyl acetate
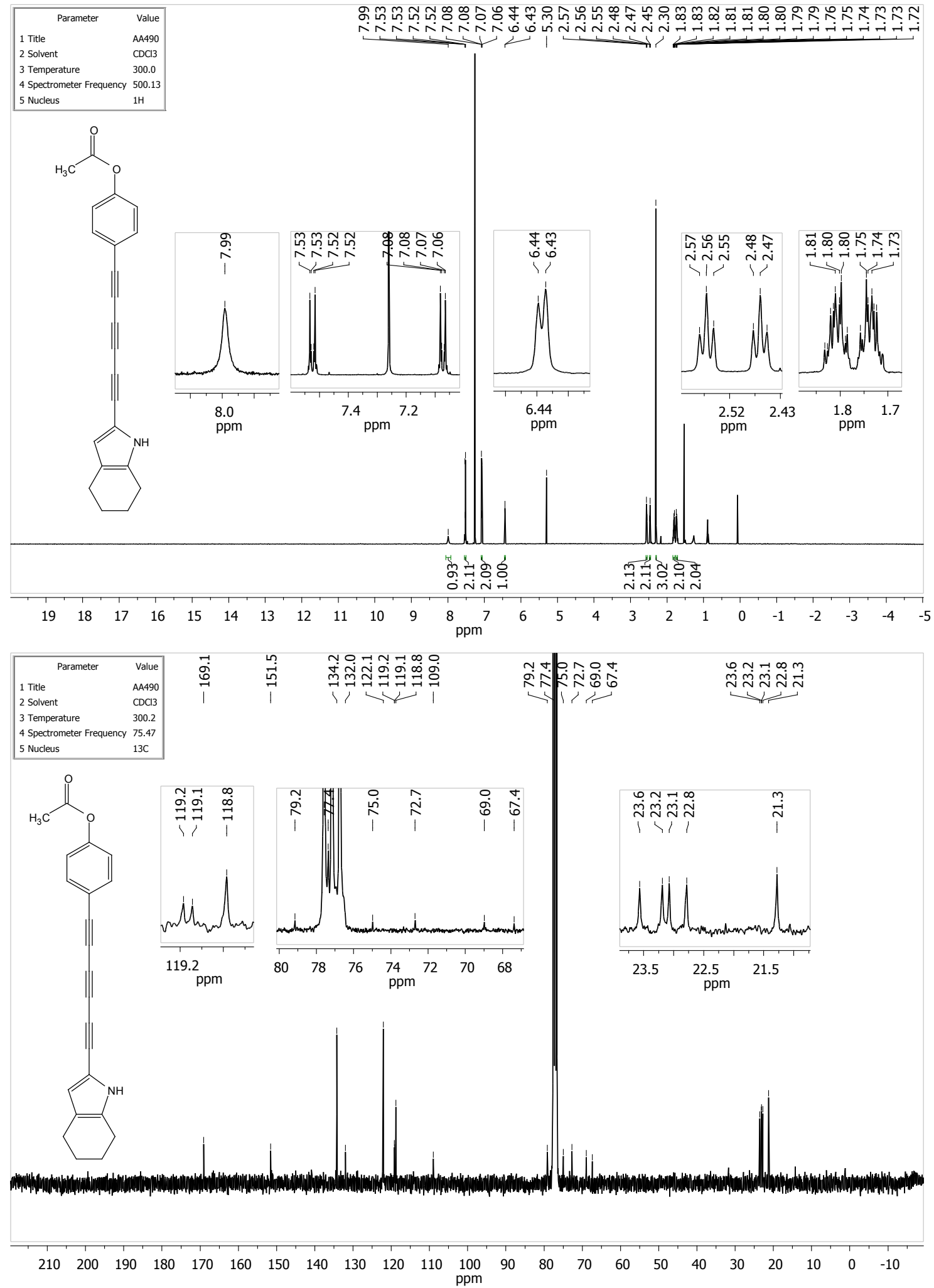


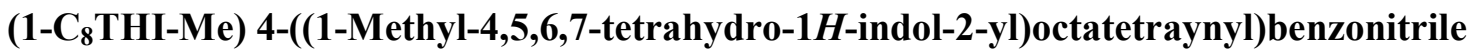

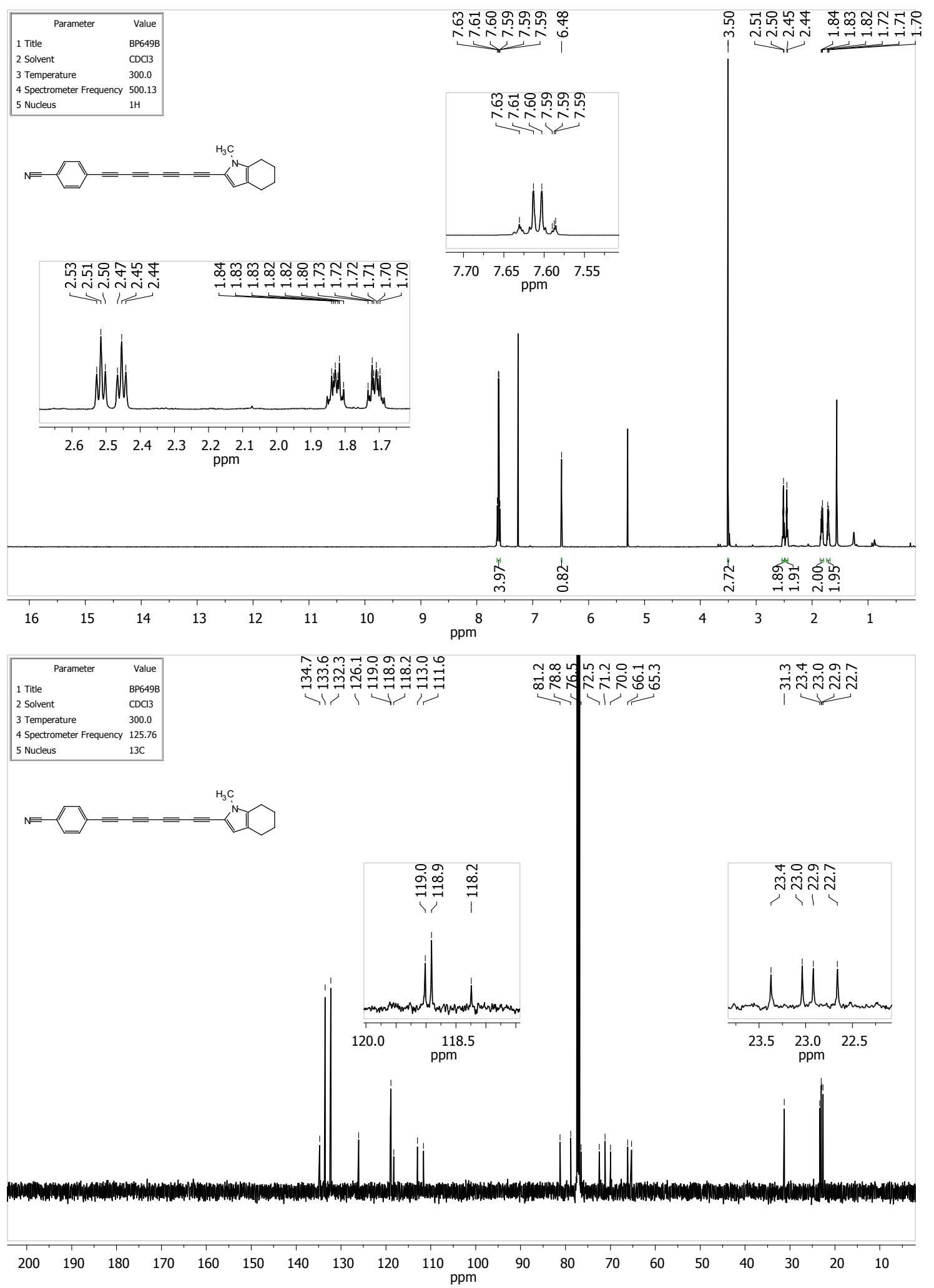


(1-C 8 THI-Vin) 4-((1-Vinyl-4,5,6,7-tetrahydro-1H-indol-2-yl)octatetraynyl)benzonitrile
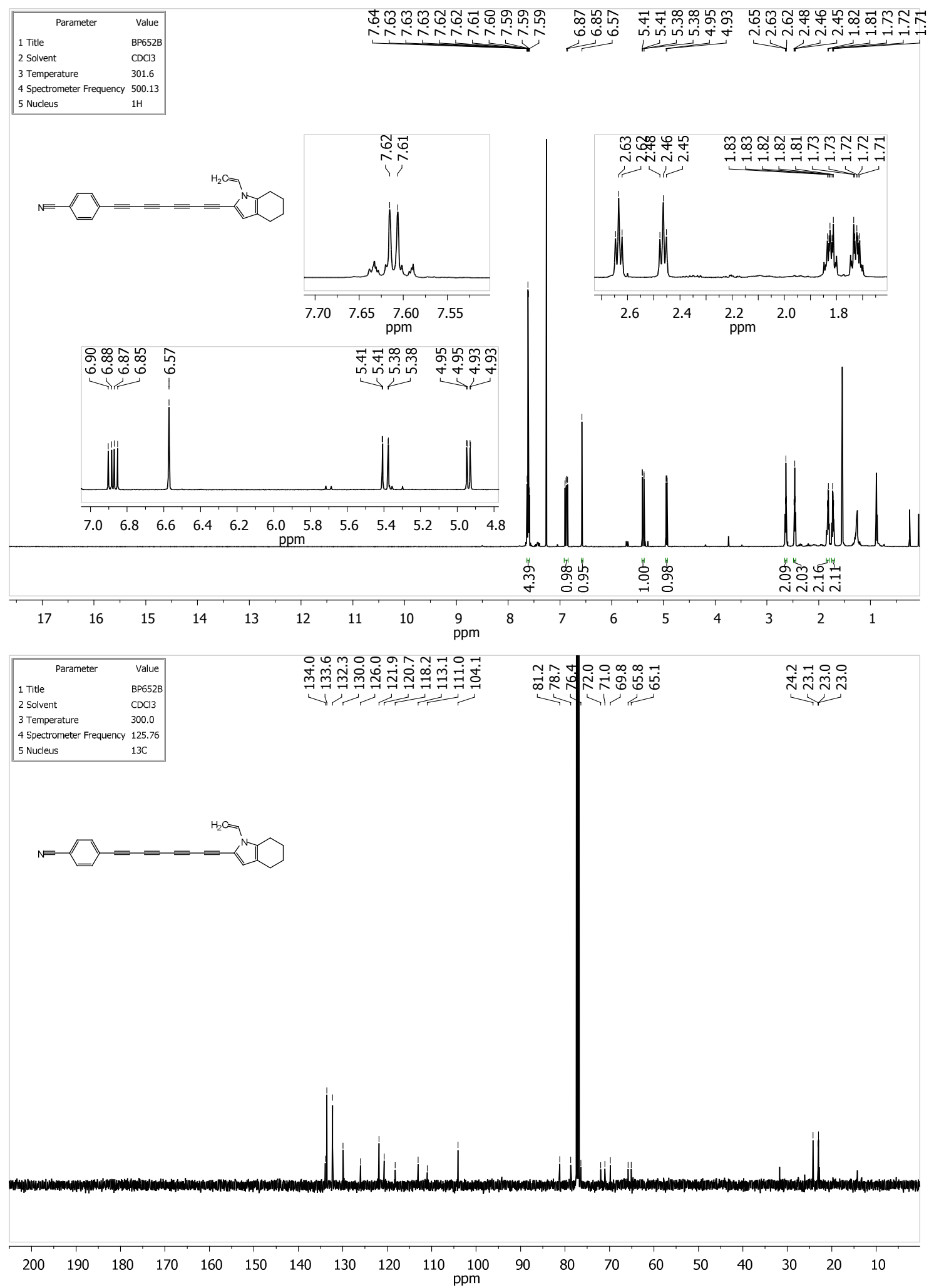


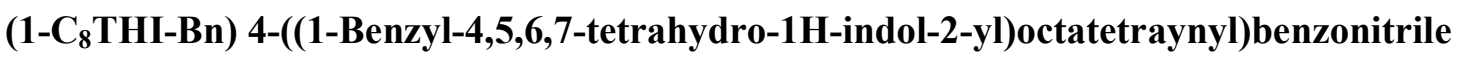
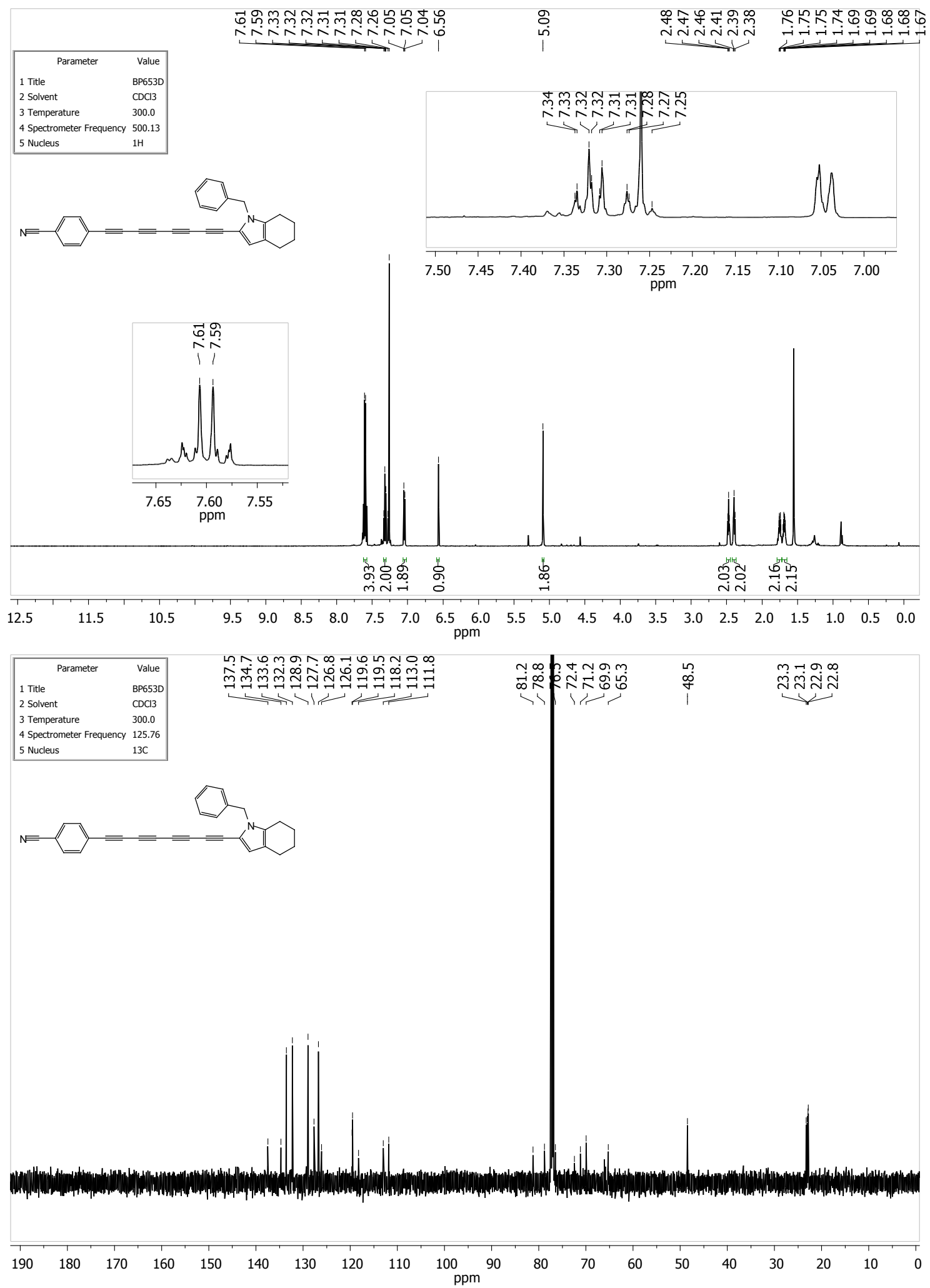
(2-C $\mathrm{C}_{8}$ THI-Me) 1-Methyl-2-((4-nitrophenyl)octatetraynyl)-4,5,6,7-tetrahydro- $1 \mathrm{H}$-indole
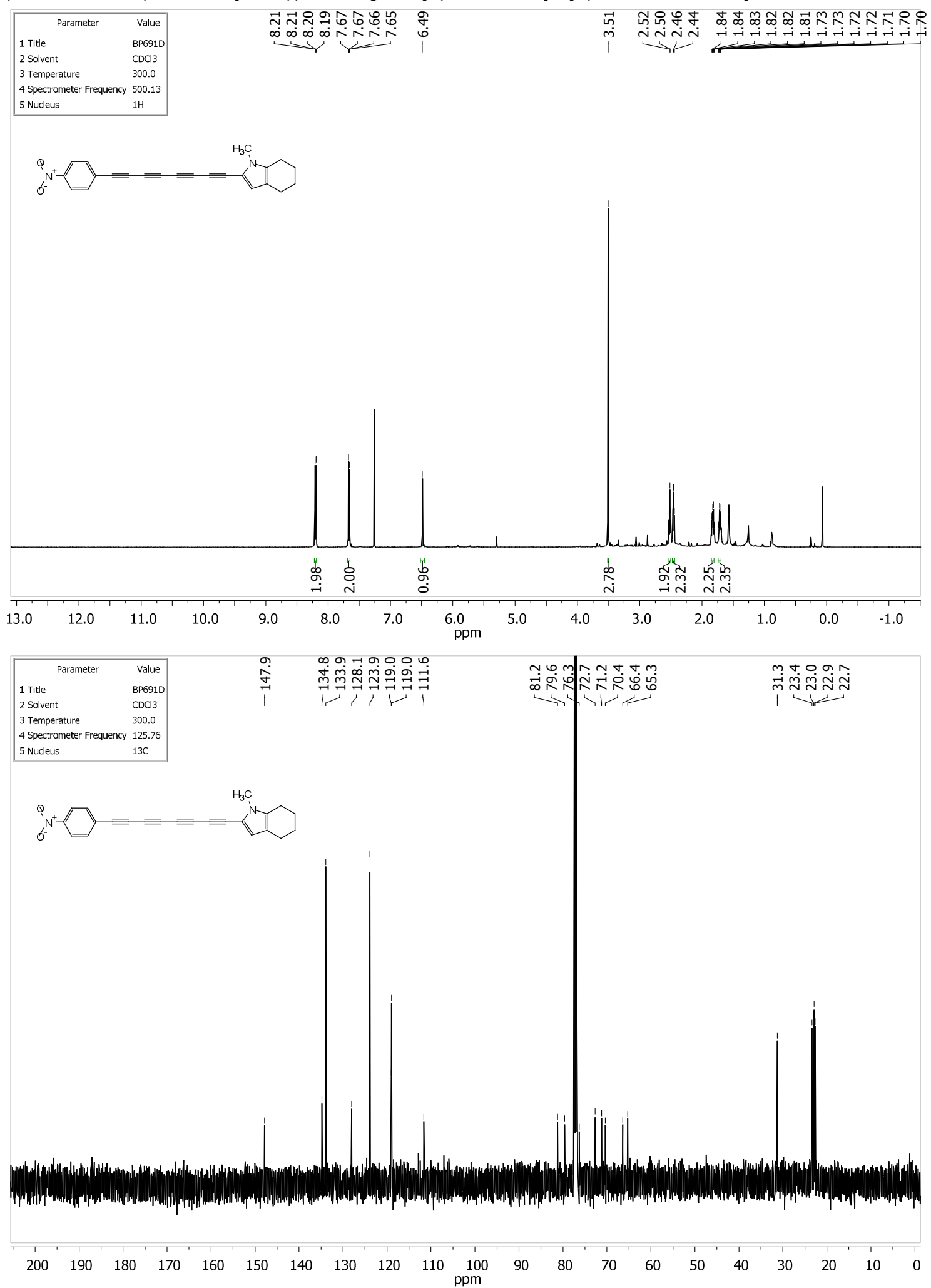
(2-C 8 THI-Vin) 2-((4-Nitrophenyl)octatetraynyl)-1-vinyl-4,5,6,7-tetrahydro-1H-indole
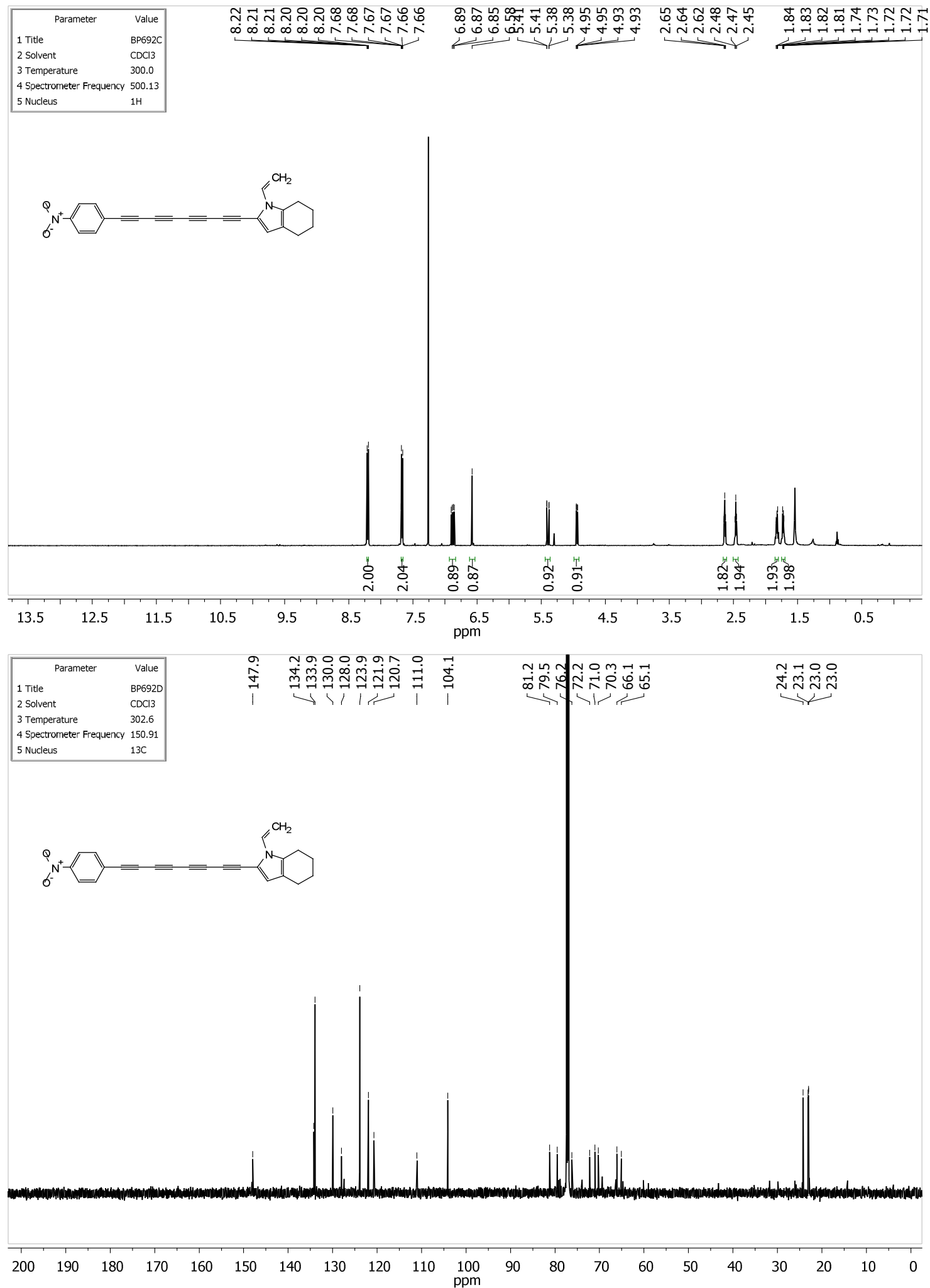
(2-C 8 THI-Bn) 2-((4-Nitrophenyl)octatetraynyl)-1-benzyl-4,5,6,7-tetrahydro-1H-indole
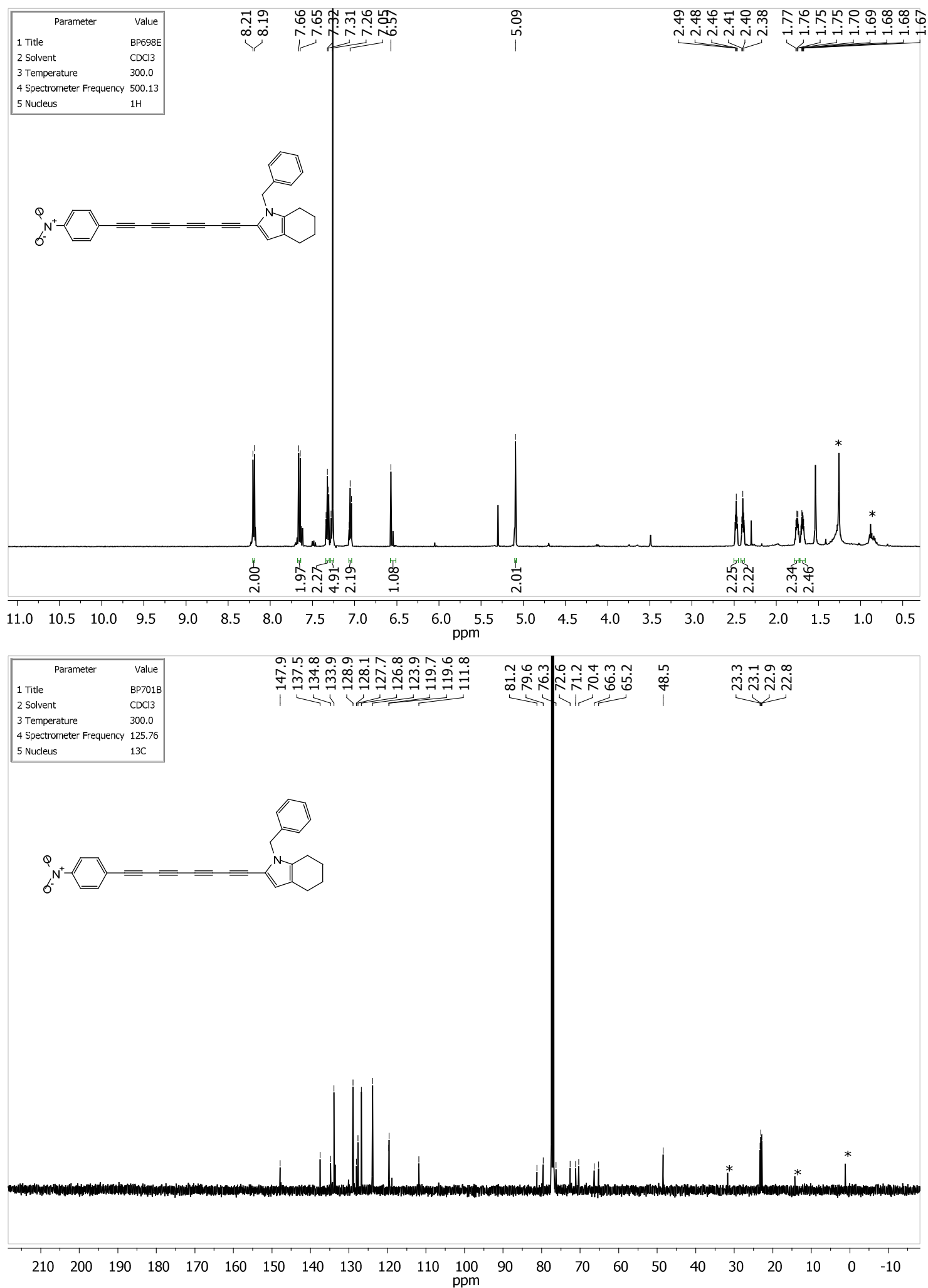